\title{
Adaptive MIMO Antenna Selection via Discrete Stochastic Optimization
}

\author{
Inaki Berenguer, Xiaodong Wang, and Vikram Krishnamurthy, Fellow, IEEE
}

\begin{abstract}
Recently it has been shown that it is possible to improve the performance of multiple-input multiple-output (MIMO) systems by employing a larger number of antennas than actually used and selecting the optimal subset based on the channel state information. Existing antenna selection algorithms assume perfect channel knowledge and optimize criteria such as Shannon capacity or various bounds on error rate. This paper examines MIMO antenna selection algorithms where the set of possible solutions is large and only a noisy estimate of the channel is available. In the same spirit as traditional adaptive filtering algorithms, we propose simulation based discrete stochastic optimization algorithms to adaptively select a better antenna subset using criteria such as maximum mutual information, bounds on error rate, etc. These discrete stochastic approximation algorithms are ideally suited to minimize the error rate since computing a closed form expression for the error rate is intractable. We also consider scenarios of timevarying channels for which the antenna selection algorithms can track the time-varying optimal antenna configuration. We present several numerical examples to show the fast convergence of these algorithms under various performance criteria, and also demonstrate their tracking capabilities.
\end{abstract}

Index Terms-Antenna selection, discrete stochastic approximation, MIMO, minimum error rate, tracking.

\section{INTRODUCTION}

$\mathbf{M}$ ULTIPLE-INPUT multiple-output (MIMO) systems can offer significant capacity gains over traditional singleinput single-output (SISO) systems [9], [34]. However, multiple antennas require multiple RF chains which consist of amplifiers, analog to digital converters, mixers, etc., that are typically very expensive. An approach for reducing the cost while maintaining the high potential data rate of a MIMO system is to employ a reduced number of RF chains at the receiver (or transmitter) and attempt to optimally allocate each chain to one of a larger number of receive (transmit) antennas which are usually cheaper elements. In this way, only the best set of antennas is used,

Manuscript received January 17, 2005; revised February 5, 2004. The work of I. Berenguer was supported in part by a Fulbright Fellowship. The work of X. Wang was supported in part by the U.S. National Science Foundation (NSF) under Grant DMS-0225692, and by the U.S. Office of Naval Research (ONR) under Grant N00014-03-1-0039. The work of V. Krishnamurthy was supported in part by NSERC and the British Columbia Advanced Systems Institute. The associate editor coordinating the review of this manuscript and approving it for publication was Dr. Jonathan H. Manton.

I. Berenguer is with the Laboratory for Communication Engineering, University of Cambridge, Cambridge CB3 OFD, U.K. (e-mail: ib226@ cam.ac.uk).

$\mathrm{X}$. Wang is with the Department of Electrical Engineering, Columbia University, New York, NY 10027 USA (e-mail: wangx@ee.columbia.edu).

V. Krishnamurthy is with the Department of Electrical Engineering, University of British Columbia, Vancouver, BC V6T 1Z4, Canada (e-mail: vikramk@ece.ubc.ca).

Digital Object Identifier 10.1109/TSP.2005.857056 while the remaining antennas are not employed, thus reducing the number of required RF chains.

Originally, antenna selection was proposed for systems having a single transmit antenna and multiple antennas at the receiver employed for standard diversity purposes at the receiver [23], [35]. Recently, for multiple transmit and multiple receive antennas several algorithms have been developed for selecting the optimal antenna subset given a particular channel realization. In [30], it is proposed to select the subset of transmit or receive antennas based on the maximum mutual information criterion and [29] gives and upper bound on the capacity of a system with antenna selection. A suboptimal algorithm that does not need to perform an exhaustive search over all possible subsets is proposed in [10] and [15]. Antenna selection algorithms that minimize the bit error rate (BER) of linear receivers in spatial multiplexing systems are presented in [18]. In [14], antenna selection algorithms are proposed to minimize the symbol error rate when orthogonal space-time block coding is used in MIMO systems. Selection algorithms that only assume knowledge of the second order statistics of the MIMO channels are also presented in [13] and [18]. Theoretical studies in [6] and [16] show that the diversity order achieved through antenna selection is the same as that of the system using the whole set of antennas in spatial multiplexing and space-time coding systems, respectively, which highly motivates the use of antenna selection.

All the algorithms appeared in the literature assume perfect channel knowledge to find the optimal antenna configuration. Moreover, these algorithms can not naturally cope with time-varying channels. This paper presents discrete stochastic approximation algorithms for selecting the optimal antenna subset based on advanced discrete stochastic optimization techniques found in the recent operations research literature [3], [4], [8]. These techniques optimize an objective function (e.g., maximum mutual information or minimum error rate) over a set of feasible parameters (e.g., antenna subsets to be used) when the objective function cannot be evaluated analytically but can only be estimated. The methods are in the same spirit as traditional adaptive filtering algorithms such as the least mean-square (LMS) algorithm in which at each iteration, the algorithms make computational simple updates to move toward a better solution and the performance gets successively improved until converging to the optimal solution. But in this case, the parameters to be optimized take discrete values (i.e., antenna indices to be used). In a similar manner to the continuous parameter case, the discrete adaptive algorithms asymptotically converge 


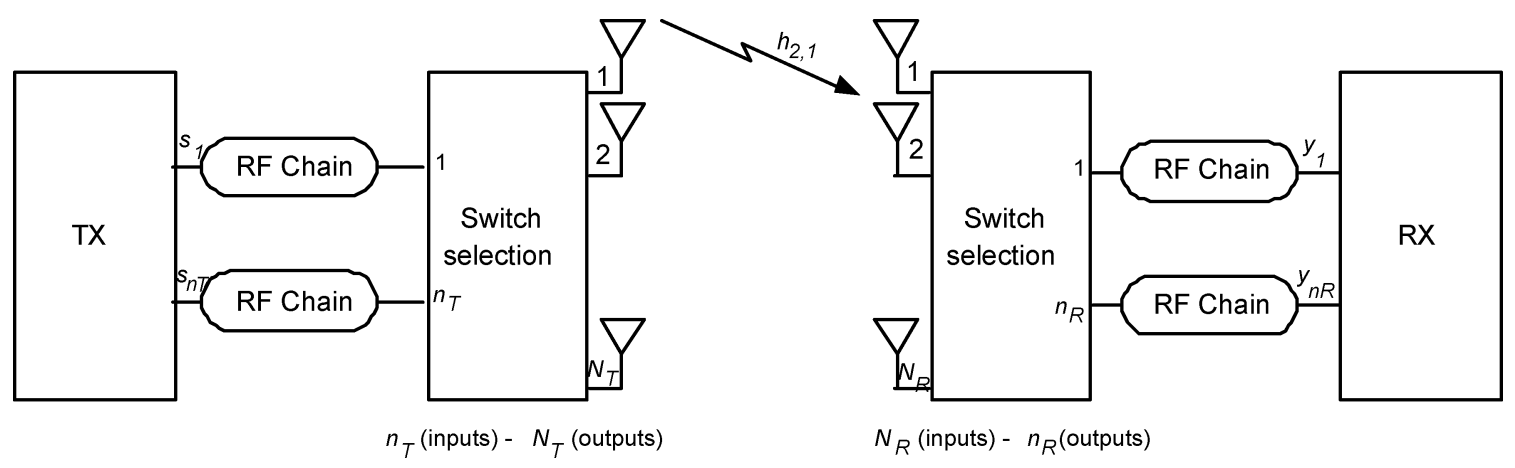

Fig. 1. Schematic representation of a MIMO system with antenna selection.

to the optimum solution. The algorithms also have an attracted property, meaning that it can be proved that they spend more time at the optimum value than at any other parameter value. In the transient phase, the algorithms converge geometrically fast toward the vicinity of the optimum point [4]. These techniques have recently been applied to solve several other problems in wireless communications [5], [24].

When the MIMO channel is time-varying, the optimal antenna subset is no longer fixed. To cope with this situation we extend our proposed algorithms to be able to track the time-varying optimal antenna configuration. The first of the proposed adaptive algorithms uses a fixed step size which acts as a forgetting factor to be able to track the optimal antenna subset. The motivation is the same as in the adaptive filtering applications with a continuous parameter space, such as LMS, in nonstationary environments where the computation is distributed over time and slow varying dynamics can be tracked. The choice of the step-size value has important effects in the tracking performance by means of convergence rate and stability. However, its value is difficult to select when the dynamics of the channel are unknown. Hence, we may optimize the tracking performance by superimposing an adaptive algorithm for the purpose of tuning the step-size parameter. Thus, we propose a second adaptive algorithm comprising two cross-coupled adaptive algorithms: 1) a discrete algorithm to adaptively select the best antenna subset and 2) a continuous algorithm to adaptively optimize the step size. Therefore, this second algorithm is attractive when the details of the underlying physical model of the MIMO channel and its variability are unknown.

The remainder of this paper is organized as follows. In Section II, the MIMO system model with antenna selection is presented. We also formulate the antenna selection problem as a discrete stochastic optimization problem. In Section III, two general discrete stochastic optimization algorithms are presented and their convergence properties are summarized. In Section IV, several antenna selection criteria are presented, including maximum mutual information, minimum bound on error rate, maximum signal-to-noise ratio, and minimum error rate. The performance of the corresponding stochastic approximation algorithms is demonstrated through several numerical examples. In Section V, antenna selection in time-varying channels is addressed. Section VI contains the conclusions.

\section{System DESCRIPTION}

\section{A. MIMO System With Antenna Selection}

Consider a MIMO system as shown in Fig. 1 with $n_{T}$ transmit and $n_{R}$ receive RF chains and suppose that there are $N_{T} \geq n_{T}$ transmit and $N_{R} \geq n_{R}$ receive antennas. The channel is represented by an $\left(N_{R} \times N_{T}\right)$ matrix $\boldsymbol{H}$ whose element $h_{i j}$ represents the complex gain of the channel between the $j$ th transmit antenna and the $i$ th receive antenna. We assume a flat fading channel remaining constant over several bursts. In this paper we concentrate on antenna selection implemented only at the receiver and therefore $N_{T}=n_{T}$. The subset of $n_{R} \leq N_{R}$ receive antennas to be employed is determined by the selection algorithm operating at the receiver which selects the optimal subset $\omega$ of all possible $\left(\begin{array}{l}N_{R} \\ n_{R}\end{array}\right)$ subsets of $n_{R}$ receive antennas. More generally, antenna selection can also be implemented at the transmitter with similar selection algorithms although the channel information needs to be known at the transmitter side. This is the case when there exists a full feedback channel so the receiver can return channel state information to the transmitter. In the case of limited feedback between the transmitter and the receiver, the selection algorithm can be implemented at the receiver and only information about the antenna indices to be used is feedback to the transmitter. Another situation where the selection algorithm is implemented at the transmitter occurs, for example, when the system employs time-division duplex (TDD) transmission so that both the uplink and downlink channels are reciprocal. In the case of antenna selection at both sides of the transmission, the same selection algorithms can be used although the amount of possible solutions, $\left(\begin{array}{l}N_{R} \\ n_{R}\end{array}\right)\left(\begin{array}{l}N_{T} \\ n_{T}\end{array}\right)$, increases dramatically. We note that loading is generally implemented when the transmitter has knowledge of the channel [31]. Therefore, if antenna selection is implemented at the transmitter, different optimality criteria will be considered to select the best antenna subset.

Denote $\boldsymbol{H}[\omega]$ as the $\left(n_{R} \times n_{T}\right)$ channel submatrix corresponding to the receive antenna subset $\omega$, i.e., rows of $\boldsymbol{H}$ corresponding to the selected antennas. The corresponding received signal is then

$$
\boldsymbol{y}=\sqrt{\frac{\rho}{n_{T}}} \boldsymbol{H}[\omega] \boldsymbol{s}+\boldsymbol{n}
$$


where $\boldsymbol{s}=\left[s_{1}, s_{2}, \ldots, s_{n_{T}}\right]^{T}$ is the $\left(n_{T} \times 1\right)$ transmitted signal vector, $\boldsymbol{y}=\left[y_{1}, y_{2}, \ldots, y_{n_{R}}\right]^{T}$ is the $\left(n_{R} \times 1\right)$ received signal vector, $\boldsymbol{n}$ is the $\left(n_{R} \times 1\right)$ received noise vector, and $\rho$ is the total signal-to-noise ratio independent of the number of transmit antennas. The entries of $\boldsymbol{n}$ are i.i.d. circularly symmetric complex Gaussian variables with unit variance, i.e., $n_{i} \sim \mathcal{N}_{c}(0,1)$. It is assumed that the transmitted symbols have unit power, i.e., $E\left\{\left|s_{i}\right|^{2}\right\}=1$.

For the problems that we are looking at in this paper, the receiver is required to estimate the channel. One way to perform channel estimation at the receiver is to use a training preamble [28]. Suppose each block of symbols comprises of $T \geq n_{T}$ MIMO training symbols $\boldsymbol{s}(1), \boldsymbol{s}(2), \ldots, \boldsymbol{s}(T)$ which are used to probe the channel. In our numerical examples we use $T=2$, $T=4$ or $T=6$. The received signals corresponding to these training symbols are

$$
\boldsymbol{y}(i)=\sqrt{\frac{\rho}{n_{T}}} \boldsymbol{H}[\omega] \boldsymbol{s}(i)+\boldsymbol{n}(i), \quad i=1,2, \ldots, T .
$$

Denote $\boldsymbol{Y}=[\boldsymbol{y}(1), \boldsymbol{y}(2), \ldots, \boldsymbol{y}(T)], \boldsymbol{S}=[\boldsymbol{s}(1), \boldsymbol{s}(2), \ldots, \boldsymbol{s}(T)]$ and $\boldsymbol{N}=[\boldsymbol{n}(1), \boldsymbol{n}(2), \ldots, \boldsymbol{n}(T)]$. Then (2) can be written as

$$
\boldsymbol{Y}=\sqrt{\frac{\rho}{n_{T}}} \boldsymbol{H}[\omega] \boldsymbol{S}+\boldsymbol{N}
$$

and the maximum likelihood estimate of the channel matrix $\boldsymbol{H}[\omega]$ is given by

$$
\begin{aligned}
\hat{\boldsymbol{H}}[\omega] & =\arg \min _{\boldsymbol{H} \in \mathbb{C}^{n_{R} \times n_{T}}}\left\|\boldsymbol{Y}-\sqrt{\frac{\rho}{n_{T}}} \boldsymbol{H} \boldsymbol{S}\right\|^{2} \\
& =\sqrt{\frac{n_{T}}{\rho}} \boldsymbol{Y} \boldsymbol{S}^{H}\left(\boldsymbol{S} \boldsymbol{S}^{H}\right)^{-1} .
\end{aligned}
$$

According to [28], the optimal training symbol sequence $\boldsymbol{S}$ that minimizes the channel estimation error should satisfy

$$
\boldsymbol{S S}^{H}=T \cdot \boldsymbol{I}_{n_{T}} .
$$

In an uncorrelated MIMO channel, the channel estimates $\hat{h}_{i, j}[\omega]$ computed using (4) with orthogonal training symbols are statistically independent Gaussian variables with [28]

$$
\hat{h}_{i, j}[\omega] \sim \mathcal{N}_{c}\left(h_{i, j}[\omega], \frac{n_{T}}{T \rho}\right) .
$$

\section{B. Problem Statement}

We now formulate the antenna selection problem as a discrete stochastic optimization problem. Denote each of the antenna subsets as $\omega=\left\{\operatorname{Ant}(1), \operatorname{Ant}(2), \ldots, \operatorname{Ant}\left(n_{R}\right)\right\}$ (e.g., selecting the first, second and sixth antennas is equivalent to $\omega=\{1,2,6\})$. Denote the set of all $P=\left(\begin{array}{l}N_{R} \\ n_{R}\end{array}\right)$ possible antenna subsets as $\Omega=\left\{\omega_{1}, \omega_{2}, \ldots, \omega_{P}\right\}$. Then, the receiver selects one of the antenna subsets in $\Omega$ to optimize a certain objective function $\Phi(\boldsymbol{H}[\omega])$ according to some specific criterion, e.g., maximum mutual information between the transmitter and the receiver, maximum signal-to-noise ratio or minimum error rate. Thus, the discrete optimization problem becomes

$$
\omega^{*}=\arg \max _{\omega \in \Omega} \Phi(\boldsymbol{H}[\omega])
$$

where we use $\omega^{*}$ to denote the global maximizer of the objective function. In practice, however, the exact value of the channel $H[\omega]$ is not available. Instead, we typically have a noisy estimate $\hat{\boldsymbol{H}}[\omega]$ of the channel.

Suppose that at time $n$ the receiver obtains an estimate of the channel, $\hat{\boldsymbol{H}}[n, \omega]$, and computes a noisy estimate of the objective function $\Phi(\boldsymbol{H}[\omega])$ denoted as $\phi[n, \omega]$. Given a sequence of i.i.d. random variables $\{\phi[n, \omega], n=1,2, \ldots\}$, if each $\phi[n, \omega]$ is an unbiased estimate of the objective function $\Phi(\boldsymbol{H}[\omega])$, then (7) can be reformulated as the following discrete stochastic optimization problem:

$$
\omega^{*}=\arg \max _{\omega \in \Omega} \Phi(\boldsymbol{H}[\omega])=\arg \max _{\omega \in \Omega} E\{\phi[n, \omega]\} .
$$

Note that existing works on antenna selection assume perfect channel knowledge and therefore treat deterministic combinatorial optimization problems. On the other hand, we assume that only noisy estimates of the channel are available and hence the corresponding antenna selection problem becomes a discrete stochastic optimization problem. In what follows, we first discuss a general discrete stochastic approximation method to solve the discrete stochastic optimization problem in (8) and then we treat different forms of the objective function under different criteria, e.g., maximum mutual information, minimum error rate, etc.

\section{Discrete Stochastic ApProximation Algorithms}

There are several methods that can be used to solve the discrete stochastic optimization problem in (8). An inefficient method to solve (8) is to compute $N$ estimates of the objective function for each of the antenna subsets $\omega \in \Omega$ and compute an empirical average which approximates the exact value of the objective function. That is, for each $\omega \in \Omega$ compute

$$
\hat{\phi}_{N}(\omega)=\frac{1}{N} \sum_{n=1}^{N} \phi[n, \omega]
$$

and then perform and exhaustive search to find $\omega^{*}=$ $\max _{\omega \in \Omega}\left\{\hat{\phi}_{N}(\omega)\right\}$. Since for any fixed $\omega \in \Omega,\{\phi[n, \omega]\}$ is an i.i.d. sequence of random variables, by the strong law of large numbers, $\hat{\phi}_{N}(\omega) \rightarrow E\{\phi[n, \omega]\}$ almost surely as $N \rightarrow \infty$. Using the finite number of antenna combinations in $\Omega$ implies that as $N \rightarrow \infty$

$$
\arg \max _{\omega \in \Omega} \hat{\phi}_{N}(\omega) \rightarrow \arg \max _{\omega \in \Omega} E\{\phi[n, \omega]\}=\arg \max _{\omega \in \Omega} \Phi(\boldsymbol{H}[\omega]) .
$$

Although the method can in principle find the optimal solution, it is highly inefficient from the antenna selection problem point of view. For each antenna subset in $\Omega, N$ estimates of the objective function would need to be computed and hence it would need to be estimated $N\left(\begin{array}{l}N_{R} \\ n_{R}\end{array}\right)$ times in total. These computations are mostly wasted in the sense that only the estimate corresponding to the optimal set $\omega^{*}$ is eventually useful. Moreover, when the channel is time-varying, this method cannot naturally track the time-varying optimum solution.

More efficient methods to solve (8) have been proposed in the operations research literature (see [4] for a survey). The ranking and selection methods, and multiple comparison methods [20] 
can be used to solve the problem. However, when the number of feasible solutions $P$ increases (usually $P>20$ antenna subsets), the complexity becomes prohibitive. More recently, a number of discrete stochastic approximation algorithms haven been proposed to solve the problem in (8), including simulated annealing type procedures [2], stochastic ruler [36], and nested partition methods [32]. In this paper, we construct iterative algorithms that resemble a stochastic approximation algorithm in the sense that they generate a sequence of estimates of the solution where each new estimate is obtained from the previous one by taking a small step in a good direction toward the global optimizer. In particular, we present two different discrete stochastic approximation algorithms based on ideas in the recent operations research literature. The most important property of the proposed algorithms is their self-learning capability-most of the computational effort is spent at the global or local optimizer of the objective function. As we will show, an attractive property of these methods is that they can be modified to track the optimum antenna subset in time-varying scenarios.

\section{A. Aggressive Discrete Stochastic Approximation Algorithm}

We now present an aggressive stochastic approximation algorithm based on [3]. We use the $P=\left(\begin{array}{l}N_{R} \\ n_{R}\end{array}\right)$ unit vectors as labels for the $P$ possible antenna subsets, i.e., $\xi=\left\{\boldsymbol{e}_{1}, \boldsymbol{e}_{2}, \ldots, \boldsymbol{e}_{P}\right\}$, where $\boldsymbol{e}_{i}$ denotes the $(P \times 1)$ vector with a one in the $i$ th position and zeros elsewhere. At each iteration, the algorithm updates the $(P \times 1)$ probability vector $\pi[n]=[\pi[n, 1], \ldots, \pi[n, P]]^{T}$ representing the state occupation probabilities with elements $\pi[n, i] \in[0,1]$ and $\sum_{i} \pi[n, i]=1$. Let $\omega^{(n)}$ be the antenna subset chosen at the $n$-th iteration. For notational simplicity, it is convenient to map the sequence of antenna subsets $\left\{\omega^{(n)}\right\}$ to the sequence $\{D[n]\} \in \xi$ of unit vectors where $\boldsymbol{D}[n]=\boldsymbol{e}_{i}$ if $\omega^{(n)}=\omega_{i}, i=1, \ldots, P$.

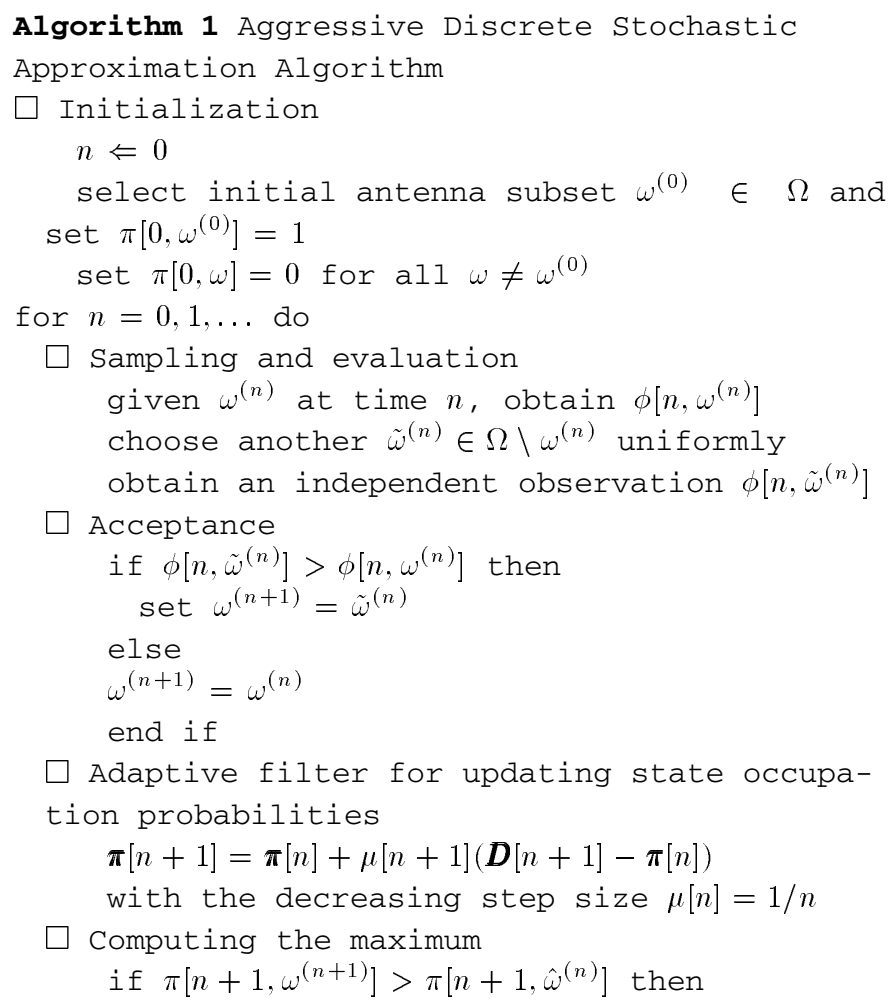

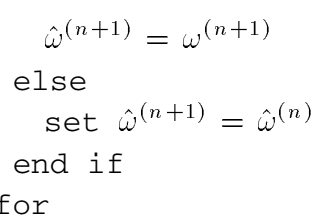

We assume that in a realistic communications scenario, each iteration of the above algorithm operates on a block of symbols comprising of $T>0$ training symbols [see description above (2)]. These $T$ training symbols are used to obtain the channel estimates $\hat{\boldsymbol{H}}\left[n, \omega^{(n)}\right]$ and hence the noisy estimate of the cost $\phi\left[n, \omega^{(n)}\right]$. In our numerical examples, we use $T=2, T=4$ or $T=6$. At the end of each iteration, antenna subset $\hat{\omega}^{(n)}$ will be selected for the next iteration.

In the Sampling and Evaluation step in Algorithm 1 , the candidate antenna subset $\tilde{\omega}^{(n)}$ is chosen uniformly from $\Omega \backslash \omega^{(n)}$. There are several variations for selecting a candidate antenna subset $\tilde{\omega}^{(n)}$. One possibility is to select a new antenna subset $\tilde{\omega}^{(n)}$ by replacing only one antenna in $\omega^{(n)}$. Define the distance $d\left(\tilde{\omega}^{(n)}, \omega^{(n)}\right)$ as the number of different antennas between the two antenna subsets $\tilde{\omega}^{(n)}$ and $\omega^{(n)}$. Hence, we can select $\tilde{\omega}^{(n)} \in \Omega \backslash \omega^{(n)}$ such that $d\left(\tilde{\omega}^{(n)}, \omega^{(n)}\right)=1$. More generally we can select a new subset $\tilde{\omega}^{(n)}$ with arbitrary distance $d\left(\tilde{\omega}^{(n)}, \omega^{(n)}\right)=D$, where $1 \leq D \leq \min \left(n_{R}, N_{R}-n_{R}\right)$. Note that any variation for selecting a candidate needs to be taken into account to prove global convergence.

To obtain the independent observations in the Sampling and Evaluation step in Algorithm 1 we proceed as follows. At time $n$, we collect training symbols to estimate the channel and compute $\phi[n, \omega]$. Now, collect other training symbols from another antenna subset and compute $\phi[n, \tilde{\omega}]$. Therefore, $\phi[n, \omega]$ and $\phi[n, \tilde{\omega}]$ are independent observations.

Remark: Heuristic variations of Sampling and Evaluation step with correlated observations. The above procedure of using independent samples to evaluate the objective function allows us to rigorously prove convergence and efficiency of the algorithm. Here, we briefly discuss three heuristic variations of the Sampling and Evaluation step that use correlated observations of the objective function. In numerical simulations, we observed that these variations also yield excellent results-however, due to the statistically correlated observations of the objective function, the proof of convergence is intractable. The first possibility is to reuse same channel observation multiple times (i.e., use the same channel estimate to compute several observations of the objective function under different antenna configurations). Another heuristic variation is to incorporate the greedy antenna selection solutions (note that this is another form of correlation) or reduce the dimension of the possible transition states (i.e., possible solutions) in the Markov chain. A third possibility is to devise hybrid solutions based on a combination of Algorithm 1 and batch processing (e.g., exhaustive search based on noisy channel estimates or greedy selections).

The sequence $\left\{\omega^{(n)}\right\}$ generated by Algorithm 1 is a Markov chain on the state space $\Omega$ which is not expected to converge and may visit each element in $\Omega$ infinitely often. On the other hand, under certain conditions the sequence $\left\{\hat{\omega}^{(n)}\right\}$ converges 
almost surely to the global maximizer $\omega^{*}$. Therefore, $\hat{\omega}^{(n)}$ can be viewed as an estimate at time $n$ of the optimal antenna subset $\omega^{*}$.

In the Adaptive filter for updating state occupation probabilities step in Algorithm 1 $\boldsymbol{\pi}[n]=[\pi[n, 1], \pi[n, 2], \ldots, \pi[n, P]]$ denotes the empirical state occupation probability at time $n$ of the Markov chain $\left\{\omega^{(n)}\right\}$. If we denote $W^{(n)}[\omega]$ for each $\omega \in \Omega$ as a counter of the number of times the Markov chain has visited antenna subset $\omega^{(n)}$ by time $n$, we can observe that $\boldsymbol{\pi}[n]=(1 / n)\left[W^{(n)}\left[\omega_{1}\right], \ldots, W^{(n)}\left[\omega_{P}\right]\right]^{T}$. Therefore, the algorithm chooses the antenna subset which has been visited most often by the Markov chain $\left\{\omega^{(n)}\right\}$ so far.

Global Convergence of Algorithm 1: A sufficient condition for Algorithm 1 to converge to the global maximizer of the objective function $\Phi(\boldsymbol{H}[\omega])$ is as follows [3]. For $\widetilde{\omega} \neq \omega^{*}, \omega \neq \omega^{*}$, and independent observations $\phi\left[n, \omega^{*}\right], \phi[n, \widetilde{\omega}], \phi[n, \omega]$

$$
\begin{aligned}
& \operatorname{Pr}\left\{\phi\left[n, \omega^{*}\right]>\phi[n, \omega]\right\}>\operatorname{Pr}\left\{\phi[n, \omega]>\phi\left[n, \omega^{*}\right]\right\}, \\
& \operatorname{Pr}\left\{\phi\left[n, \omega^{*}\right]>\phi[n, \widetilde{\omega}]\right\}>\operatorname{Pr}\{\phi[n, \omega]>\phi[n, \widetilde{\omega}]\} .
\end{aligned}
$$

It is shown in [3] that if the conditions (11) and (12) are satisfied, the sequence $\left\{\omega^{(n)}\right\}$ is an homogeneous irreducible and aperiodic Markov chain with state space $\Omega$. Moreover, the sequence $\left\{\hat{\omega}^{(n)}\right\}$ converges almost surely to $\omega^{*}$ in the sense that the Markov chain $\left\{\omega^{(n)}\right\}$ spends more time in $\omega^{*}$ than any other state. The transition kernel for the Markov chain $\left\{\omega^{(n)}\right\}$ is given by a transition probability matrix $K$ whose elements are given by

$$
\begin{aligned}
k_{i, j} & =\operatorname{Pr}\left\{\omega^{(n+1)}=\omega_{j} \mid \omega^{(n)}=\omega_{i}\right\} \\
& =\frac{1}{|\Omega|-1} \operatorname{Pr}\left\{\phi\left[n, \omega_{j}\right]>\phi\left[n, \omega_{i}\right]\right\}
\end{aligned}
$$

for all $i, j \in\{1, \ldots, P\}, i \neq j$, and

$$
\begin{aligned}
k_{i, i} & =1-\sum_{j \in\{1, \ldots, P\}, j \neq i} k_{i, j} \\
& =\frac{1}{|\Omega|-1} \sum_{j \in\{1, \ldots, P\}, j \neq i} \operatorname{Pr}\left\{\phi\left[n, \omega_{j}\right] \leq \phi\left[n, \omega_{i}\right]\right\}
\end{aligned}
$$

for all $i \in\{1, \ldots, P\}$ (assuming that the observations $\phi[n, \omega]$ are independent for all $n$ and $\omega$ ).

The two conditions in (11) and (12) basically state the conditions that the Markov transition matrix defined in (13) and (14) need to satisfy. Condition (11) states that $k_{j, i}>k_{i, j}$ for $\omega_{i}=\omega^{*}$ and $\omega_{j} \neq \omega^{*}$, i.e., it is more probable for the Markov chain to move into a state corresponding to $\omega^{*}$ from a state that does not correspond to $\omega^{*}$ than in the other direction. And condition (12) states that $k_{j, i}>k_{j, \ell}$ for $\omega_{i}=\omega^{*}$ and $\omega_{\ell} \neq \omega^{*}, \omega_{j} \neq \omega^{*}$, i.e., once the Markov chain is in a state that does not correspond to $\omega^{*}$, it is more probable to move into a state that corresponds to $\omega^{*}$ than into any other state.

Thus Algorithm 1 is a globally convergent algorithm that spends more time at the global optimum than any other value. This property of spending more time at the global optimum than any other value is called the "attraction" property of the algorithm in [4]. It means that the algorithm is efficient-see also the classic paper [11] for details on how to compare the asymptotic efficiency of simulation based estimators.
An important issue regarding proving the convergence of Algorithm 1 is to choose estimators that satisfy properties (11) and (12). Next, we propose a conservative algorithm that converges to the global optimizer of the objective function under less restrictive conditions.

\section{B. Conservative Discrete Stochastic Approximation Algorithm}

Now, we present a conservative discrete stochastic approximation algorithm based on ideas in [8] with less restrictive conditions for global convergence.

Algorithm 2 Conservative Discrete Stochastic Approximation Algorithm

Initialization

$n \Leftarrow 0$

select initial antenna subset $\omega^{(0)} \in \Omega$

Initialize $P$-dimensional vectors $\boldsymbol{h}[0], l[0]$ and $\overline{\boldsymbol{k}}[0]$ to zero

for $n=0,1, \ldots$ do

$\square$ Sampling, evaluation and update choose another $\tilde{\omega}^{(n)} \in \Omega \backslash \omega^{(n)}$ uniformly obtain an independent observation $\phi\left[n, \tilde{\omega}^{(n)}\right]$ and update:

$\boldsymbol{l}\left[n+1, \tilde{\omega}^{(n)}\right]=\boldsymbol{l}\left[n, \tilde{\omega}^{(n)}\right]+\phi\left[n, \tilde{\omega}^{(n)}\right] \quad$ (Accumulated $\overline{\boldsymbol{k}}\left[n+1, \phi\left[n, \tilde{\omega}^{(n)}\right]\right] \quad=\quad \overline{\boldsymbol{k}}\left[n, \phi\left[n, \tilde{\omega}^{(n)}\right]\right]+$

1 (Occupation time)

$\boldsymbol{h}\left[n, \phi\left[n, \tilde{\omega}^{(n)}\right]\right]=\boldsymbol{l}\left[n+1, \phi\left[n, \tilde{\omega}^{(n)}\right]\right] / \overline{\boldsymbol{k}}[n+$

$\left.1, \phi\left[n, \tilde{\omega}^{(n)}\right]\right]$ (Average cost vector)

$\square$ Acceptance

if $\boldsymbol{h}\left[n, \tilde{\omega}^{(n)}\right]>\boldsymbol{h}\left[n, \omega^{(n)}\right]$ then

set $\omega^{(n+1)}=\tilde{\omega}^{(n)}$

else

$\omega^{(n+1)}=\omega^{(n)}$

end if

Update estimate of optimum subset $\hat{\omega}^{(n+1)}=\omega^{(n+1)}$

end for

As in the Adaptive filter step of Algorithm 1, the Sampling, evaluation and update step in Algorithm 2 can be rewritten as an adaptive algorithm with a decreasing step size as: update the occupation time diagonal matrix $\boldsymbol{K}[\cdot]$ as $\boldsymbol{K}[n+1]=$ $\boldsymbol{K}[n]+\mu[n+1]\left(\boldsymbol{d i a g}\left(\boldsymbol{D}_{1}[n], \ldots, \boldsymbol{D}_{P}\right)-\boldsymbol{K}[n]\right)$ and

$$
\begin{aligned}
& \boldsymbol{h}[n+1]=\boldsymbol{h}[n]+\mu[n+1] \boldsymbol{K}^{-1}[n+1] \\
& \quad \times\left(\phi\left[n, \tilde{\omega}^{(n)}\right] \boldsymbol{D}[n]-\operatorname{diag}\left(\boldsymbol{D}_{1}[n], \ldots, \boldsymbol{D}_{P}\right) \boldsymbol{h}[n]\right)
\end{aligned}
$$

where as in Algorithm $1, \mu[n]=1 / n, D[n]=e_{i}$ if $\tilde{\omega}^{(n)}=\omega_{i}$ and $\boldsymbol{D}_{\boldsymbol{i}}[n]$ represents the $i$ th component of $\boldsymbol{D}[n]$. The $(P \times$ $P$ )-dimensional matrix $\boldsymbol{K}[n]$ in (15) is initialized to $\boldsymbol{K}[0]=\boldsymbol{I}_{P}$. The conservative name refers to the convergence of the Markov chain $\left\{\omega^{(n)}\right\}$ in Algorithm 2 since the sequence $\left\{\omega^{(n)}\right\}$ in Algorithm 1 is not expected to converge-in Algorithm 1 only $\left\{\hat{\omega}^{(n)}\right\}$ converges. Note that in Algorithm 2, we only require one estimate of the objective function per iteration and in general, the complexity is similar to the one of Algorithm 1. 
Global Convergence of Algorithm 2: As proved in [8], a sufficient condition for Algorithm 2 to converge to the global optimum is to use unbiased observations of the objective function.

\section{ANTENNA SELECTIONS UNDER DifFERENT CRITERIA}

In this section, we use the optimization algorithms to optimize four different objective functions $\Phi(\boldsymbol{H}[\omega])$. These are: 1) MIMO mutual information; 2) bounds on error rate; 3 ) SNR; and 4) error rate. Simulation results are provided in each case to demonstrate the performance of the corresponding stochastic approximation algorithm.

\section{A. Maximum MIMO Mutual Information}

Assuming that the channel matrix $\boldsymbol{H}[\omega]$ is known at the receiver, but not at the transmitter, the mutual information between the transmitter and receiver is given by [9] and [34]

$$
\mathcal{I}[\omega]=\log \operatorname{det}\left(\boldsymbol{I}_{n_{T}}+\frac{\rho}{n_{T}} \boldsymbol{H}^{H}[\omega] \boldsymbol{H}[\omega]\right) \mathrm{bit} / \mathrm{s} / \mathrm{Hz} .
$$

One criterion for selecting the antennas is to maximize the above mutual information, i.e., choosing the objective function $\Phi(\boldsymbol{H}[\omega])=\mathcal{I}[\omega]$.

1) Aggressive Algorithm to Optimize the Mutual Information: We now present an implementation of Algorithm 1 to find the maximum of the mutual information in (16) using

$$
\phi[n, \omega]=\log \operatorname{det}\left(\boldsymbol{I}_{n_{T}}+\frac{\rho}{n_{T}} \hat{\boldsymbol{H}}[n, \omega]^{H} \hat{\boldsymbol{H}}[n, \omega]\right) .
$$

Notes on Convergence: To prove the convergence to the global optimum when we use (17) in Algorithm 1, we need to verify that conditions (11) and (12) are satisfied. We propose the following result that will help us to verify these conditions.

Proposition 1: The random variable in (17) can be accurately approximated by the Gaussian distribution

$$
\phi[n, \omega] \sim \mathcal{N}\left(\mu_{\mathcal{I}_{\omega}}, \sigma_{\mathcal{I}_{\omega}}^{2}\right)
$$

where

$$
\begin{aligned}
\mu_{\mathcal{I}_{\omega}} & =E_{\hat{\boldsymbol{H}}}\left\{\log \operatorname{det}\left(\boldsymbol{I}_{n_{T}}+\frac{\rho}{n_{T}} \hat{\boldsymbol{H}}[n, \omega]^{H} \hat{\boldsymbol{H}}[n, \omega]\right)\right\} \\
& =\frac{e^{\operatorname{tr}(\Lambda[\omega])}}{\ln (2)\left(\Gamma(t-s+1)^{s} \operatorname{det}(\boldsymbol{V})\right.} \sum_{k=1}^{s} \operatorname{det}(\Psi(k))
\end{aligned}
$$

with $\operatorname{det}(\Psi(k)), k=1, \ldots, s$ are $(s \times s)$ matrices with entries

$$
\{\Psi(k)\}_{i, j}=\left\{\begin{array}{lr}
\int_{0}^{\infty} y^{t-i} \ln (1+\alpha y) e^{-y} & j=k \\
\times{ }_{0} F_{1}\left(t-s+1, y \lambda_{j}[\omega]\right) d y, & \\
\Gamma(t-i+1) & \\
\times{ }_{1} F_{1}\left(t-i+1, t-s+1, \lambda_{j}[\omega]\right), & j \neq k
\end{array}\right.
$$

where $t=\max \left(n_{T}, n_{R}\right)$ and $s=\min \left(n_{T}, n_{R}\right), \alpha=\rho / n_{T}$. $n_{T} / \rho T=1 / T, 0<\lambda_{1}[\omega]<\lambda_{2}[\omega]<\ldots<\lambda_{s}[\omega]<\infty$ are the nonzero ordered eigenvalues of $\left(n_{T} / T \rho\right) \boldsymbol{H}^{H} \boldsymbol{H}, \Lambda[\omega]=$ $\operatorname{diag}\left(\lambda_{1}[\omega], \ldots, \lambda_{s}[\omega]\right), \boldsymbol{V}$ is an $(s \times s)$ matrix with determinant $\operatorname{det}(\boldsymbol{V})=\Pi_{1 \leq i<j \leq s}\left(\lambda_{i}[\omega]-\lambda_{j}[\omega]\right),{ }_{0} F_{1}(\cdot, \cdot)$ is the generalized hypergeometric function defined in [17, Eq. (9.14.1)] as ${ }_{0} F_{1}(a, z)=\sum_{k=0}^{\infty}\left(z^{k} /(a)_{k} k !\right), \Gamma(\cdot)$ is the gamma function [17, Eq. (8.31.1)], ${ }_{1} F_{1}(\cdot, \cdot, \cdot)$ is the confluent hypergeometric function [17, Eq. (9.210.1)] defined as

$$
{ }_{1} F_{1}(a ; b ; z)=\sum_{k=0}^{\infty} \frac{(a)_{k}}{(b)_{k}} \frac{z^{k}}{k !}
$$

and $(a)_{k}=a(a+1) \ldots(a+k-1)$ is the Pochhammer symbol. The second moment of the estimator is

$$
E\left\{\hat{\mathcal{I}}_{\omega}^{2}\right\}=\frac{e^{\operatorname{tr}(\Lambda[\omega])}}{\ln ^{2}(2)\left(\Gamma(t-s+1)^{s} \operatorname{det}(\boldsymbol{V})\right.} \sum_{k=1}^{s} \sum_{l=1}^{s} \operatorname{det}(\Psi(k, l))
$$

where $\operatorname{det}(\Psi(k, l)), k, l=1, \ldots, s$ are $(s \times s)$ matrices with entries as shown in (23), at the bottom of the page, and the variance in (18) can be computed as

$$
\sigma_{\mathcal{I}_{\omega}}^{2}=E\left\{\hat{\mathcal{I}}_{\omega}^{2}\right\}-\mu_{\mathcal{I}_{\omega}}^{2} .
$$

Proof: The channel estimate in (17) with orthogonal training symbols in (5) contains independent elements $\hat{h}_{i j[\omega]}=\mathcal{N}_{c}\left(h_{i j}[\omega], n_{T} / T \rho\right)$. Therefore, the channel estimate can be written as $\hat{\boldsymbol{H}}[n, \omega]=\boldsymbol{H}[\omega]+\Delta \boldsymbol{H}[n, \omega]$ which contains a constant term $\boldsymbol{H}[\omega]$ and a random complex Gaussian matrix $\Delta \boldsymbol{H}[n, \omega]$ of zero mean. Then, the estimate of the mutual information function can be written as

$$
\begin{aligned}
\phi[n, \omega]=\log \operatorname{det}\left(\boldsymbol{I}_{n_{T}}+\frac{\rho}{n_{T}}\right. & (\boldsymbol{H}[\omega]+\Delta \boldsymbol{H}[n, \omega])^{H} \\
& \times(\boldsymbol{H}[\omega]+\Delta \boldsymbol{H}[n, \omega]))
\end{aligned}
$$

which is equivalent to the mutual information of a Rician flat fading MIMO channel with a nonzero mean matrix $\boldsymbol{H}[\omega]$. The expressions of the mean and variance of the capacity of a noniid Rician are derived in [22] which correspond to (19) and (24), respectively.

In particular, under the maximum mutual information criterion, we note that the estimator in (17) has a positive bias, i.e., $\mu_{\mathcal{I}_{\omega}}>\mathcal{I}[\omega]$ in (16). This fact can be also understood with the results in [22] and the parallelism of the estimate of the mutual information computed with noisy channel estimates and capacity results of the Rician channel. Moreover, it has been observed that although the estimator is biased

if $\mathcal{I}\left[\omega_{i}\right]>\mathcal{I}\left[\omega_{k}\right]$, then $\mu_{\mathcal{I}_{\omega_{i}}}>\mu_{\mathcal{I}_{\omega_{k}}}, \forall i, k \in\{1, \ldots, P\}$.

To prove the convergence to the global optimum using (17) in Algorithm 1, we still need to verify that conditions (11) and (12) are satisfied. Consider three different antenna subsets $\omega_{i}=\omega^{*}$

$$
\{\Psi(k)\}_{i, j}= \begin{cases}\int_{0}^{\infty} y^{t-i} \ln ^{2}(1+\alpha y) e^{-y} F_{1}\left(t-s+1, y \lambda_{j}[\omega]\right) d y, & j=k=l \\ \int_{0}^{\infty} y^{t-i} \ln (1+\alpha y) e^{-y} F_{1}\left(t-s+1, y \lambda_{j}[\omega]\right) d y, & j=k \text { or } j=l ; k \neq l \\ \Gamma(t-i+1)_{1} F_{1}\left(t-i+1, t-s+1, \lambda_{j}[\omega]\right), & j \neq k ; j \neq l\end{cases}
$$


and $\omega_{l}, \omega_{j} \in\left\{\Omega \backslash \omega^{*}\right\}$. From (17) and (18), we have independent random variables $\phi\left[n, \omega_{i}\right] \sim \mathcal{N}\left(\mu_{\mathcal{I}_{\omega_{i}}}, \sigma_{\mathcal{I}_{\omega_{i}}}^{2}\right), \phi\left[n, \omega_{j}\right] \sim$ $\mathcal{N}\left(\mu_{\mathcal{I}_{\omega_{j}}}, \sigma_{\mathcal{I}_{\omega_{j}}}^{2}\right)$ and $\phi\left[n, \omega_{l}\right] \sim \mathcal{N}\left(\mu_{\mathcal{I}_{\omega_{l}}}, \sigma_{\mathcal{I}_{\omega_{l}}}^{2}\right)$. Condition (11) can be written as

$$
\operatorname{Pr}\left(\phi\left[n, \omega_{i}\right]-\phi\left[n, \omega_{j}\right]>0\right)>\operatorname{Pr}\left(\phi\left[n, \omega_{j}\right]-\phi\left[n, \omega_{i}\right]>0\right)
$$

and since samples of $\phi$ are independent and Gaussian distributed, (27) is equivalent to

$$
\begin{aligned}
\operatorname{Pr}(\mathcal{N} & \left.\left(\mu_{\mathcal{I}_{\omega_{i}}}-\mu_{\mathcal{I}_{\omega_{j}}}, \sigma_{\mathcal{I}_{\omega_{i}}}^{2}+\sigma_{\mathcal{I}_{\omega_{j}}}^{2}\right)>0\right) \\
& >\operatorname{Pr}\left(\mathcal{N}\left(\mu_{\mathcal{I}_{\omega_{j}}}-\mu_{\mathcal{I}_{\omega_{i}}}, \sigma_{\mathcal{I}_{\omega_{j}}}^{2}+\sigma_{\mathcal{I}_{\omega_{i}}}^{2}\right)>0\right) .
\end{aligned}
$$

From (26), we have $\max \left\{\mu_{\mathcal{I}_{\omega_{i}}}, \mu_{\mathcal{I}_{\omega_{j}}}, \mu_{\mathcal{I}_{\omega_{l}}}\right\}=\mu_{\mathcal{I}_{\omega_{i}}}$ which implies $\left(\mu_{\mathcal{I}_{\omega_{i}}}-\mu_{\mathcal{I}_{\omega_{j}}}\right)>\left(\mu_{\mathcal{I}_{\omega_{j}}}-\mu_{\mathcal{I}_{\omega_{i}}}\right)$. Therefore, (27) holds since both terms have the same variance. Consider now condition (12). We can express it as

$$
\begin{aligned}
\operatorname{Pr}\left(\mathcal{N}\left(\mu_{\mathcal{I}_{\omega_{i}}}-\mu_{\mathcal{I}_{\omega_{j}}}, \sigma_{\mathcal{I}_{\omega_{i}}}^{2}+\sigma_{\mathcal{I}_{\omega_{j}}}^{2}\right)>0\right) \\
\quad>\operatorname{Pr}\left(\mathcal{N}\left(\mu_{\mathcal{I}_{\omega_{l}}}-\mu_{\mathcal{I}_{\omega_{j}}}, \sigma_{\mathcal{I}_{\omega_{l}}}^{2}+\sigma_{\mathcal{I}_{\omega_{j}}}^{2}\right)>0\right)
\end{aligned}
$$

which can be rewritten as

$$
\operatorname{Pr}\left(\phi\left[n, \omega_{i}\right]-\phi\left[n, \omega_{j}\right]>0\right)>\operatorname{Pr}\left(\phi\left[n, \omega_{l}\right]-\phi\left[n, \omega_{j}\right]>0\right)
$$

that is equivalent to

$$
\frac{\mu_{\mathcal{I}_{\omega_{i}}}-\mu_{\mathcal{I}_{\omega_{j}}}}{\sqrt{\sigma_{\mathcal{I}_{\omega_{i}}}^{2}+\sigma_{\mathcal{I}_{\omega_{j}}}^{2}}}>\frac{\mu_{\mathcal{I}_{\omega_{l}}}-\mu_{\mathcal{I}_{\omega_{j}}}}{\sqrt{\sigma_{\mathcal{I}_{\omega_{l}}}^{2}+\sigma_{\mathcal{I}_{\omega_{j}}}^{2}}}
$$

The inequality in (31) has been observed to hold after extensive simulations using the expressions of the mean and the variance given in (19) and (24), respectively.

2) Conservative Algorithm to Optimize the Mutual Information: We now present an implementation of the conservative algorithm and prove that it converges to the global maximum of the mutual information in (16). Since the logarithm is a monotonically increasing function, the antenna subset $\omega^{*}$ maximizing $\log \operatorname{det}(\cdot)$ is identical to that maximizing $\operatorname{det}(\cdot)$.

In the Sampling, evaluation and update step of Algorithm 2 choose

$$
\phi[n, \omega]=\operatorname{det}\left(\boldsymbol{I}_{n_{T}}+\frac{\rho}{n_{T}} \hat{\boldsymbol{H}}_{1}^{H}[n, \omega] \hat{\boldsymbol{H}}_{2}[n, \omega]\right)
$$

where the channel estimates $\hat{\boldsymbol{H}}_{1}[n, \omega]$ and $\hat{\boldsymbol{H}}_{2}[n, \omega]$ are obtained from independent training blocks. We consider the case in which $\hat{\boldsymbol{H}}_{1}[n, \omega]$ and $\hat{\boldsymbol{H}}_{2}[n, \omega]$ satisfy (6).

Theorem 1: With $\phi[n, \omega]$ computed according to (32), the sequence $\left\{\hat{\omega}^{(n)}\right\}$ generated by Algorithm 2 converges to the antenna subset $\omega^{*}$ corresponding to the global maximizer of the MIMO mutual information in (16).

Proof: To prove global convergence, we only need to show that $\phi[n, \omega]$ of (32) is unbiased, which is proved in Appendix A.

To reduce the training symbols needed to estimate the channel in Algorithm 2, in practical systems we can use a single sample of the channel $\hat{\boldsymbol{H}}_{1}[n, \omega]$ and choose

$$
\phi[n, \omega]=\operatorname{det}\left(\boldsymbol{I}_{n_{T}}+\frac{\rho}{n_{T}} \hat{\boldsymbol{H}}_{1}[n, \omega]^{H} \hat{\boldsymbol{H}}_{1}[n, \omega]\right) .
$$

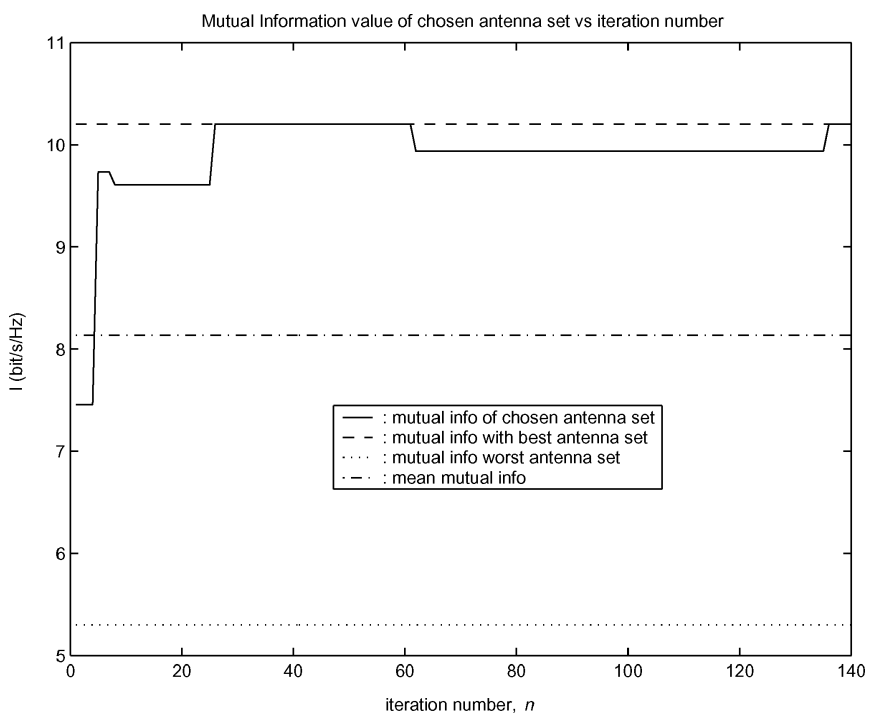

Fig. 2. Single run of Algorithm 1: mutual information value of the chosen antenna subset versus iteration number $n$.

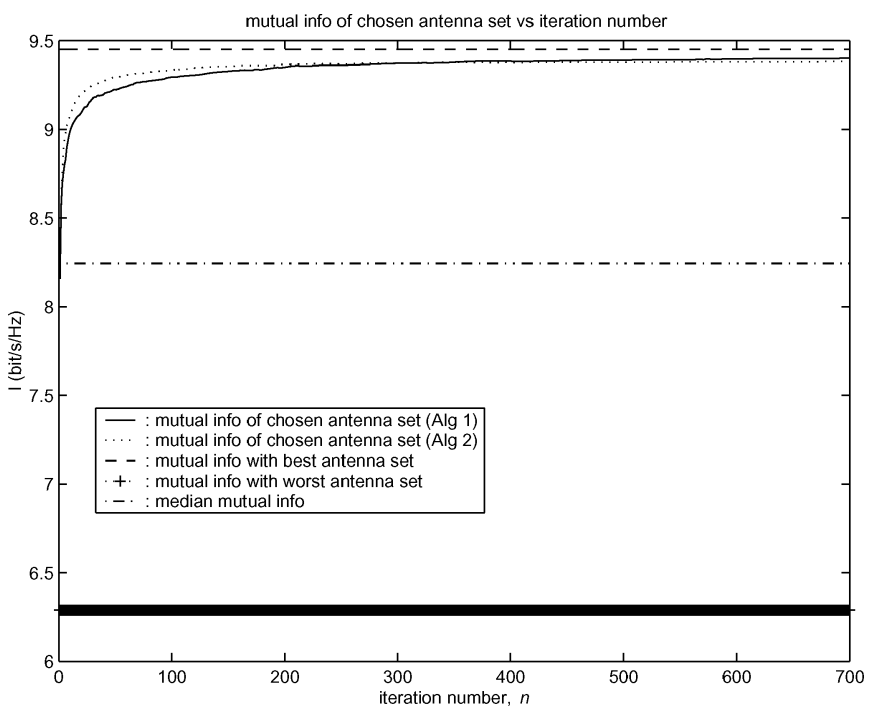

Fig. 3. Average of the mutual information values of chosen antenna subsets by Algorithm 1 and Algorithm 2 (over 3000 runs) versus iteration number $n$.

Although this sample is biased, numerical results can show that Algorithm 2 still converges to the global optimum.

3) Simulation Results: We consider the performance of Algorithm 1 which selects the antenna subset maximizing the MIMO mutual information using (33) as an estimate of the objective function. We consider $n_{T}=2, N_{R}=8$ and $n_{R}=4$ antennas. We use the ML channel estimate in (4) with $T=4$ orthogonal training symbols to estimate the channel. We set $\rho=10 \mathrm{~dB}$. The $\left(N_{R} \times n_{T}\right)$ channel $\boldsymbol{H}$ is randomly generated and fixed during the whole simulation. The initial antenna subset was randomly selected. Fig. 2 shows one run of the algorithm. In the same figure we show the mutual information of the best antenna subset and the worst antenna subset, as well as the median mutual information among the $\left(\begin{array}{l}8 \\ 4\end{array}\right)=70$ antenna configurations, found by exhaustive search. Next, in Fig. 3 we consider 700 iterations per execution and we average the mutual information of the antenna subset selected at all 


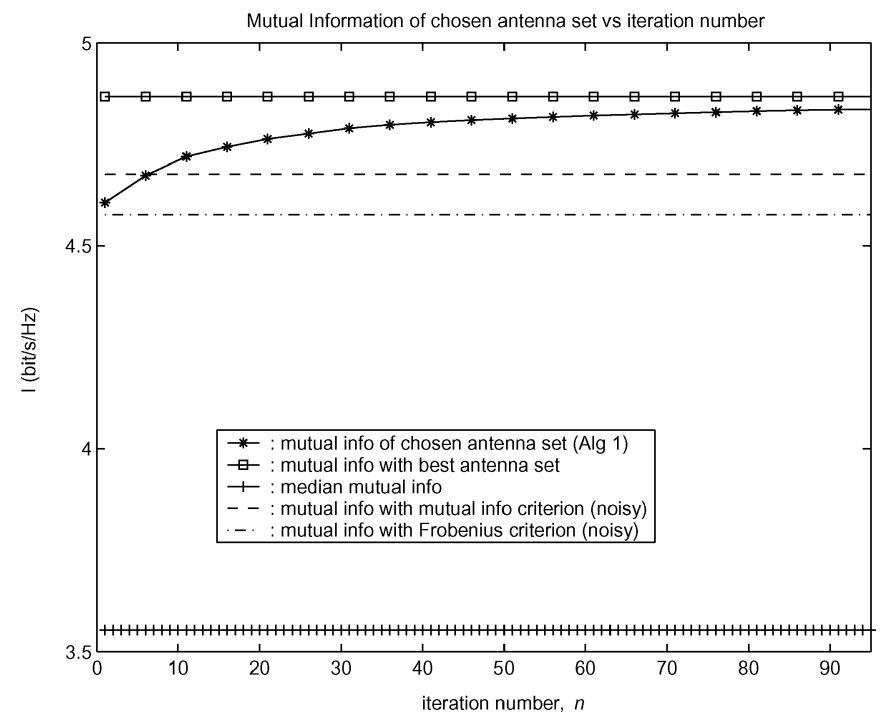

Fig. 4. Average of the mutual information values of chosen antenna subsets versus iteration number with a hot start adaptive algorithm.

iterations over 1000 channel realizations. In the same figure we also show the performance of Algorithm 2. It is seen that in the transient phase, Algorithm 2 has slightly better convergence behavior than Algorithm 1 although in the long term, Algorithm 1 performs better. From both figures, it is seen that the algorithms adaptively move to the best antenna subset. We observe that although the algorithms take some time to converge to the optimal antenna subset, they move very fast to an antenna subset inducing high MIMO mutual information.

From a practical point of view, instead of initializing the algorithm by choosing a random antenna subset, there are several variations for selecting $\omega^{(0)}$ to avoid the initial transient phase (i.e., hot start initialization). For instance, based on a noisy channel estimate, select the antenna subset whose matrix $\hat{\boldsymbol{H}}\left[0, \omega^{(0)}\right]$ has maximum Frobenius norm (i.e., select the antennas that receive maximum power). Consider a system with $N_{R}=5, n_{T}=n_{R}=2, T=4$, and SNR $=6 \mathrm{~dB}$. Fig. 4 shows the average mutual information over 100 initial channel realizations versus the iteration number with the hot start adaptive algorithm based on the maximum Frobenius norm initial selection. It is seen that from the very first iteration the adaptive algorithm is close to the optimal solution. In the same figure we show the mutual information of the antenna subset selected based on the maximum mutual information criterion found by exhaustive search using noisy channel estimates.

\section{B. Minimum Bounds on Error Rate}

Consider the system in Fig. 1 where the transmitted data $\boldsymbol{s}$ is multiplexed into the $n_{T}$ transmit antennas. The input-output relationship is expressed in (1) where in this case, the transmitted symbols $s_{i}$ belong to a finite constellation $\mathcal{A}$ of size $|\mathcal{A}|$. The receive antennas see the superposition of all transmitted signals. The task of the receiver is to recover the transmitted data $s$. The ML detection rule is given by

$$
\hat{\boldsymbol{s}}=\arg \min _{\boldsymbol{s} \in \mathcal{A}^{n_{T}}}\left\|\boldsymbol{y}-\sqrt{\frac{\rho}{n_{T}}} \boldsymbol{H}[\omega] \boldsymbol{s}\right\|^{2} .
$$

At high signal-to-noise ratio, we can upper bound the probability of error of the ML detector using the union bound [18] which is a function of the squared minimum distance $d_{\min , r}^{2}$ of the received constellation given by [19]

$$
d_{\min , r}^{2}[\omega]=\min _{\substack{\boldsymbol{s}_{i}, \boldsymbol{s}_{j} \in \mathcal{A}^{n_{T}} \\ \boldsymbol{s}_{i} \neq \boldsymbol{s}_{j}}}\left\|\boldsymbol{H}[\omega]\left(\boldsymbol{s}_{i}-\boldsymbol{s}_{j}\right)\right\|^{2} .
$$

Therefore, minimizing the union bound on error probability is equivalent to maximizing $d_{\min , r}^{2}$. In Algorithm 1, we use $\phi[n, \omega]=\min _{\substack{\boldsymbol{s}_{i}, \boldsymbol{s}_{j} \in \mathcal{A}^{n_{T}} \\ \boldsymbol{s}_{i} \neq \boldsymbol{s}_{j}}}\left\|\hat{\boldsymbol{H}}[n, \omega]\left(\boldsymbol{s}_{i}-\boldsymbol{s}_{j}\right)\right\|^{2}$. In Algorithm 2, we propose the following theorem.

Theorem 2: With

$$
\phi[n, \omega]=\min _{\substack{\boldsymbol{s}_{i}, \boldsymbol{s}_{j} \in \mathcal{A}^{n_{T}} \\ \boldsymbol{s}_{i} \neq \boldsymbol{s}_{j}}}\left[\hat{\boldsymbol{H}}_{1}[n, \omega]\left(\boldsymbol{s}_{i}-\boldsymbol{s}_{j}\right)\right]^{H}\left[\hat{\boldsymbol{H}}_{2}[n, \omega]\left(\boldsymbol{s}_{i}-\boldsymbol{s}_{j}\right)\right]
$$

the sequence $\left\{\hat{\omega}^{(n)}\right\}$ generated by Algorithm 2 converges to the global maximizer $\omega^{*}$ of (35).

Proof: Applying similar arguments to the proof of Theorem 1 it follows that the estimate of the objective function in (36) satisfies the requirements of global convergence specified by Algorithm 2.

To reduce the number of required training symbols in the implementation of Algorithm 2, we can use a biased estimator of $d_{\min , r}^{2}[\omega]$ using only one estimate of the channel as in Algorithm 1 .

Note that the computation of $d_{\min , r}^{2}[\omega]$ is performed over $|\mathcal{A}|^{n_{T}}\left(|\mathcal{A}|^{n_{T}}-1\right)$ possibilities for each antenna subset which can be prohibitive for large $|\mathcal{A}|$ or $n_{T}$. Let $\lambda_{\min }[\omega]$ be the smallest singular value of $\boldsymbol{H}[\omega]$ and let the minimum squared distance of the transmit constellation be $d_{\min , t}^{2}=\min _{\boldsymbol{s}_{i}, \boldsymbol{s}_{j} \in \mathcal{A}^{n_{T}}}\left\|\left(\boldsymbol{s}_{i}-\boldsymbol{s}_{j}\right)\right\|^{2}$. Then, it is shown in [19] that $d_{\min , r}^{2}[\omega] \geq \lambda_{\min }^{2}[\omega] d_{\min , t}^{2}$. Therefore, a selection criterion can be simplified to select the antenna subset $\omega \in \Omega$ whose associated channel matrix $\boldsymbol{H}[\omega]$ has the largest minimum singular value. In our problem, based on an estimate of the channel at time $n$, we let $\phi[n, \omega]=\hat{\lambda}_{\min }[n, \omega]$.

Simulation Results: We consider the performance of Algorithm 1 with $N_{R}=10, n_{R}=2$ (45 different antenna subsets) and $n_{T}=2$ with ML channel estimate and $T=2$ orthogonal training symbols to estimate the channel. The channel $\boldsymbol{H}$ is assumed to be fixed during the whole run of the algorithm and we set $\rho=10 \mathrm{~dB}$. We compare three antenna configuration: 1) best antenna set: antenna set with $\left.\max _{\omega_{i}}\left(\lambda_{\min }\left[\omega_{i}\right]\right) ; 2\right)$ worst antenna set: antenna set with $\min _{\omega_{i}}\left(\lambda_{\min }\left[\omega_{i}\right]\right)$; and 3$)$ the antenna set chosen by the algorithm at iteration $n$, i.e., $\hat{\omega}^{(n)}$. Antenna sets 1) and 2) are found by an exhaustive search assuming that the channel is perfectly known. We performed 90 iterations of the algorithm. Fig. 5 shows a single run of the algorithm. Fig. 6 shows the average of 100 runs of the algorithm over the same fixed channel $\boldsymbol{H}$. It is observed from the two figures that the algorithm converges and as in the maximum of the mutual information case, it is seen that although it takes some time to converge, it moves quite fast to an antenna subset whose channel has a high $\lambda_{\min }$. It is important to point out that Algorithm 1 using the above cost functions converges to the antenna subset which maximizes $d_{\min , r}$ or $\lambda_{\min }$. However, these criteria 


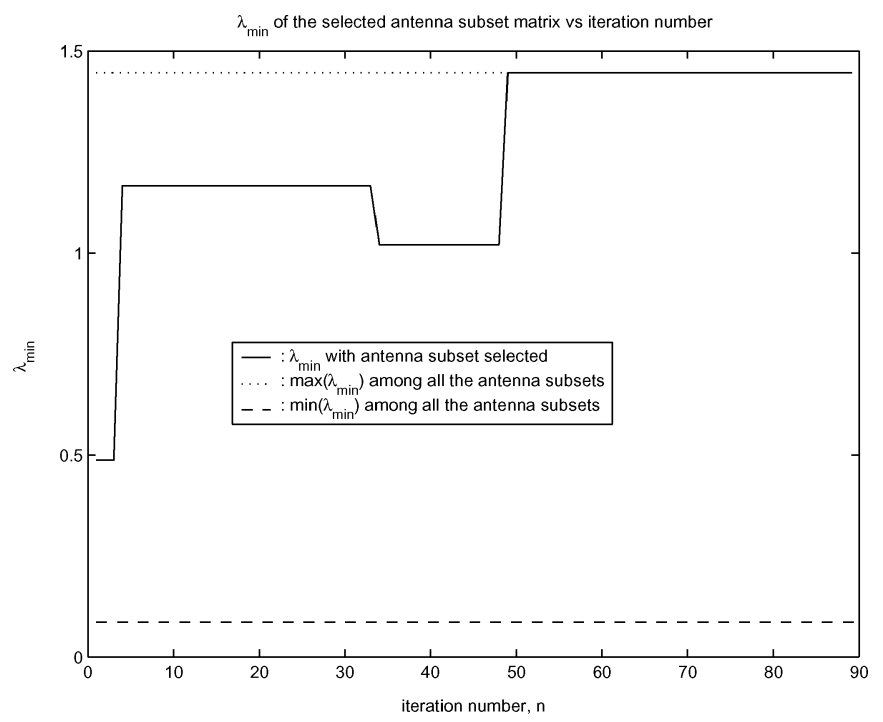

Fig. 5. Single run of Algorithm 1: minimum singular value of the antenna subset selected versus iteration number $n$.

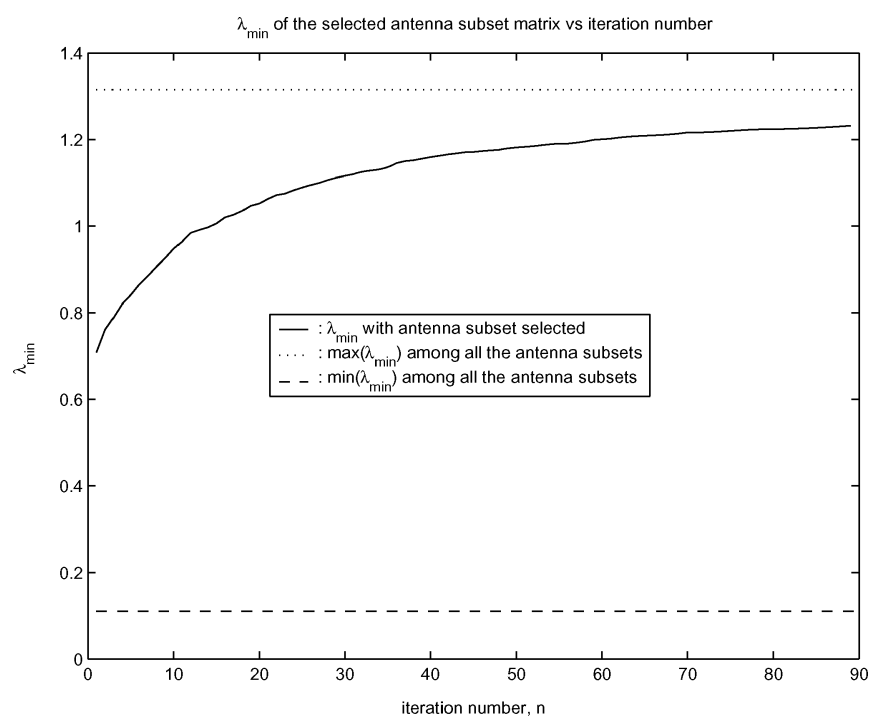

Fig. 6. Average (over 100 runs) of the minimum singular value of the channel of the chosen antenna subsets versus iteration number $n$.

do not necessarily minimize the bit error probability since they are based on bounds. Actually, we can show situations in which both cases converge to different antenna subsets and none of them correspond to the antenna subset minimizing the bit error probability. The main reason for this is that the bound is tight only for high signal-to-noise ratio. To observe this phenomenon we consider a system with $N_{R}=10, n_{R}=2, n_{T}=2$, and $\rho=10 \mathrm{~dB}$. We average the BER of 30 different channels realizations $\boldsymbol{H}$ and with each channel realization and each antenna subset within the same channel we send 14000 QPSK symbols to compute the BER. Performing an exhaustive search (assuming perfect knowledge of the channel), we find the antenna subsets under each criterion. We observe that with the $\mathcal{I}_{\max }$ criterion, the antenna subset selected obtains a BER of 0.00054 , with the $\lambda_{\min }$ criterion the BER is 0.00049 , with the $d_{\min , r}$ criterion the BER is 0.00039 , and the minimum BER of all antenna subsets is 0.00035 .

\section{Maximum SNR}

Linear receivers for the system in (1) are simpler receivers in which the received vector $\boldsymbol{y}$ is linearly transformed to obtain

$$
\boldsymbol{z}=\boldsymbol{G} \boldsymbol{y}=\sqrt{\frac{\rho}{n_{T}}} \boldsymbol{G H}[\omega] \boldsymbol{s}+\boldsymbol{G n} .
$$

For linear receivers, the symbol error probability is influenced by the post processing signal-to-noise ratio. For the minimum mean-square error (MMSE) receiver, after applying the equalizer matrix $\boldsymbol{G}=\sqrt{\rho / n_{T}}\left(\left(\rho / n_{T}\right) \boldsymbol{H}^{H}[\omega] \boldsymbol{H}[\omega]+\boldsymbol{I}_{n_{T}}\right)^{-1} \boldsymbol{H}^{H}[\omega]$ the signal-to-noise ratio for each of the $n_{T}$ transmitted data streams can be expressed as [18]

$$
\begin{array}{r}
\mathrm{SNR}_{i}^{(M M S E)}[\omega]=\frac{1}{\left(\frac{\rho}{n_{T}} \boldsymbol{H}^{H}[\omega] \boldsymbol{H}[\omega]+\boldsymbol{I}_{n_{T}}\right)_{i i}^{-1}}-1 \\
\text { for } i=1, \ldots, n_{T} .
\end{array}
$$

Correspondingly, in Algorithm 1 we set

$$
\phi[n, \omega]=\max _{i \in\left[1, n_{T}\right]}\left(\frac{\rho}{n_{T}} \hat{\boldsymbol{H}}^{H}[n, \omega] \hat{\boldsymbol{H}}[n, \omega]+\boldsymbol{I}_{n_{T}}\right)_{i i}^{-1} .
$$

For the zero-forcing (ZF) receiver, $\boldsymbol{G}=\sqrt{n_{T} / \rho} \boldsymbol{H}^{\dagger}[\omega]$, where $\dagger$ denotes the pseudo-inverse. For each of the $n_{T}$ transmitted data streams, the signal-to-noise ratio after applying the equalizer matrix $\boldsymbol{G}$ can be expressed as [18]

$$
\operatorname{SNR}_{i}^{(Z F)}[\omega]=\frac{\rho}{n_{T}\left(\boldsymbol{H}^{H}[\omega] \boldsymbol{H}[\omega]\right)_{i i}^{-1}}, \quad \text { for } \quad i=1, \ldots, n_{T}
$$

and correspondingly, in Algorithm 1 we use

$$
\phi[n, \omega]=\max _{i \in\left[1, n_{T}\right]}\left(\hat{\boldsymbol{H}}^{H}[n, \omega] \hat{\boldsymbol{H}}[n, \omega]\right)_{i i}^{-1} .
$$

Another case of interest is when the orthogonal space-time block codes are employed. Using the coding and decoding algorithms in [1], [33], the receiver signal-to-noise ratio of the data stream is given by [14]

$$
\operatorname{SNR}[\omega]=\frac{\rho}{n_{T}} \operatorname{trace}\left(\boldsymbol{H}^{H}[\omega] \boldsymbol{H}[\omega]\right)=\frac{\rho}{n_{T}}\|\boldsymbol{H}[\omega]\|_{F}^{2}
$$

where $\|\cdot\|_{F}^{2}$ indicates the Frobenius norm. Therefore, in Algorithm 1 we may use $\phi[n, \omega]=\|\hat{\boldsymbol{H}}[n, \omega]\|_{F}^{2}$. With Algorithm 2, we propose the following theorem to obtain an unbiased estimate of the objective function.

Theorem 3: With

$$
\phi[n, \omega]=\operatorname{trace}\left[\hat{\boldsymbol{H}}_{1}^{H}[n, \omega] \hat{\boldsymbol{H}}_{2}^{H}[n, \omega]\right]
$$

the sequence $\left\{\hat{\omega}^{(n)}\right\}$ generated by Algorithm 2 converges to the global maximizer of (42). 
Proof: Applying similar arguments to the proof of Theorem 1 it follows that the estimate of the objective function in (43) satisfies the requirements of global convergence specified by Algorithm 2.

\section{Minimum Error Rate}

As shown in Section IV-B for the ML receiver, the antenna subset chosen by the different criteria based on bounds do not necessarily choose the antenna subset minimizing the bit error rate (BER). In this section, we propose an antenna selection algorithm that directly minimizes the symbol or bit error rate of the system under any type of receivers.

In the proposed method, a noisy estimate of the simulated error rate is used as the cost function in the stochastic approximation algorithm instead of a noisy estimate of a bound. The method proceeds as follows. Assume for example that the ML decoding algorithm in (34) is used. At time $n$, estimate the channel $\hat{\boldsymbol{H}}[n, \omega]$ with antenna subset $\omega$. At the receiver, generate $m$ fake random symbol vectors $\boldsymbol{S}_{f}=\left[\boldsymbol{s}_{f}(1), \ldots, \boldsymbol{s}_{f}(m)\right]$ with $s_{f, k}(i) \in \mathcal{A}$ and perform a simulation of the form

$$
\boldsymbol{Y}_{f}=\sqrt{\frac{\rho}{n_{T}}} \hat{\boldsymbol{H}}[n, \omega] \boldsymbol{S}_{f}+\boldsymbol{N}
$$

where the $\left(n_{R} \times m\right)$ matrix $\boldsymbol{N}$ contains i.i.d. $\mathcal{N}_{c}(0,1)$ samples. Perform the ML detection on (44) to obtain

$$
\hat{\boldsymbol{S}}_{f}=\arg \min _{\boldsymbol{S} \in \mathcal{A}^{n} T^{\times m}}\left\|\boldsymbol{Y}_{f}-\sqrt{\frac{\rho}{n_{T}}} \hat{\boldsymbol{H}}[n, \omega] \boldsymbol{S}\right\|^{2}
$$

and estimate the bit error rate $\widehat{\mathrm{BER}}[n, \omega]$ by comparing $\hat{\boldsymbol{S}}_{f}$ and $\boldsymbol{S}_{f}$. In this way, at time $n$, an estimate of the real BER $[\omega]$ has been obtained. Note that the noise in the estimate of the BER is due to the error in the estimate of the channel and to the limitation in the number of fake symbols used in the simulations. The number of fake symbol vectors required to obtain a good estimate of the BER depends on the signal-to-noise ratio $\rho$ of the channel. For low signal-to-noise ratio, only short fake sequences are needed. The estimated BER will become more accurate as we increase the number $m$ of the fake symbols although the complexity of the algorithm will grow accordingly. Therefore, in Algorithm 1 we use $\phi[n, \omega]=-\widehat{\mathrm{BER}}[n, \omega]$ as an observation of the cost function.

Note that the fake symbols $\boldsymbol{s}_{f}$ are not actually sent through the channel. They are merely generated at the receiver to estimate the BER. It is important to point that this method uses an estimate of the BER and a closed-form BER expression is not needed, which makes it appealing for other receivers for which even a tight bound is difficult to find. Among these receivers, we may cite the ordered nulling and cancellation BLAST receivers [12]. Obviously, the same method can be used in antenna selection for MIMO systems employing various space-time coding schemes. Moreover, it is straightforward to modify the algorithm to minimize the symbol error rate or frame error rate as well.

The main disadvantage of this approach is that in the high SNR regime, the BER can be very low and therefore, a large amount of fake symbols need to be used if we want to obtain a

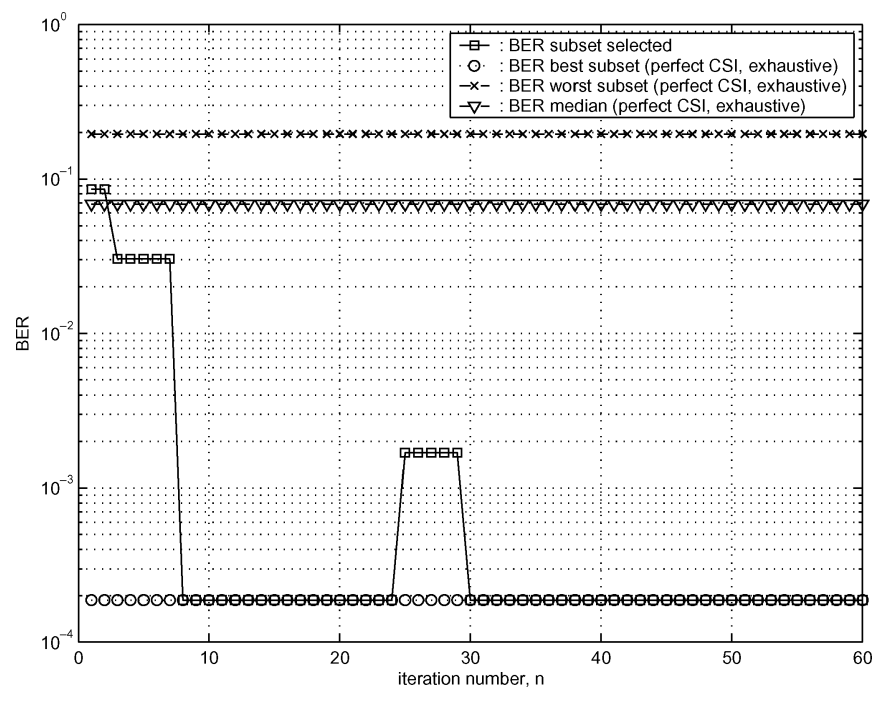

Fig. 7. Single run of Algorithm 1: BER of the of the chosen antenna subset versus iteration number $n$ employing an ML receiver.

good estimate of the BER. On the other hand, it has been observed by simulations that the antenna subset having the minimum BER at a SNR value $\rho_{1}$, corresponds to the antenna subset having the minimum BER for a range of SNR values around $\rho_{1}$ as long as there is not a large difference in the SNR. Therefore, we can reduce the SNR of the simulation to find the best antenna subset when the SNR is high. In this way, a smaller number of fake symbols will be needed to obtain a good estimate of the error rate and the complexity can be considerably reduced.

Simulation Results: To show the performance of this method in Algorithm 1 we consider first an ML receiver. We use QPSK symbols and we consider $N_{R}=6, n_{R}=2$ (i.e., 15 different antenna configurations) and $n_{T}=2$. The $\left(N_{R} \times n_{T}\right)$ channel $\boldsymbol{H}$ is randomly generated and fixed during the whole simulation. We set $\rho=9 \mathrm{~dB}$ and we use $T=6$ orthogonal training symbols to estimate the channel. Before starting the algorithm, long simulations are performed assuming perfect channel knowledge over all antenna configurations to find the BER associated with each antenna subset (including the worst and best antenna subset). We run $n=60$ iterations of the algorithm with $m=500$ fake symbols per iteration. Fig. 7 shows the BER of the antenna selected by the algorithm comparing it with the median, the best and the worst BER. It is seen that the algorithm converges to the optimal antenna subset. Moreover, it is observed that antenna selection at the receiver can improve the BER by more than two orders of magnitude with respect to the median BER even for such small values of the signal-to-noise ratio in the channel.

Now, we consider the performance of this method in a system employing the ordered nulling and cancellation BLAST receiver. We consider the MMSE criterion for the nulling operation [12]. We use the same channel realization and system parameters as in the ML case. Before starting the algorithm, long simulations are performed assuming perfect channel knowledge over all antenna configurations to find the BER associated with each antenna subset. We use 400 fake symbols per iteration. Fig. 8 shows the BER of the antenna selected by the algorithm and we compare it with the median, the best and the worst BER. As in the ML case, it is seen that the 


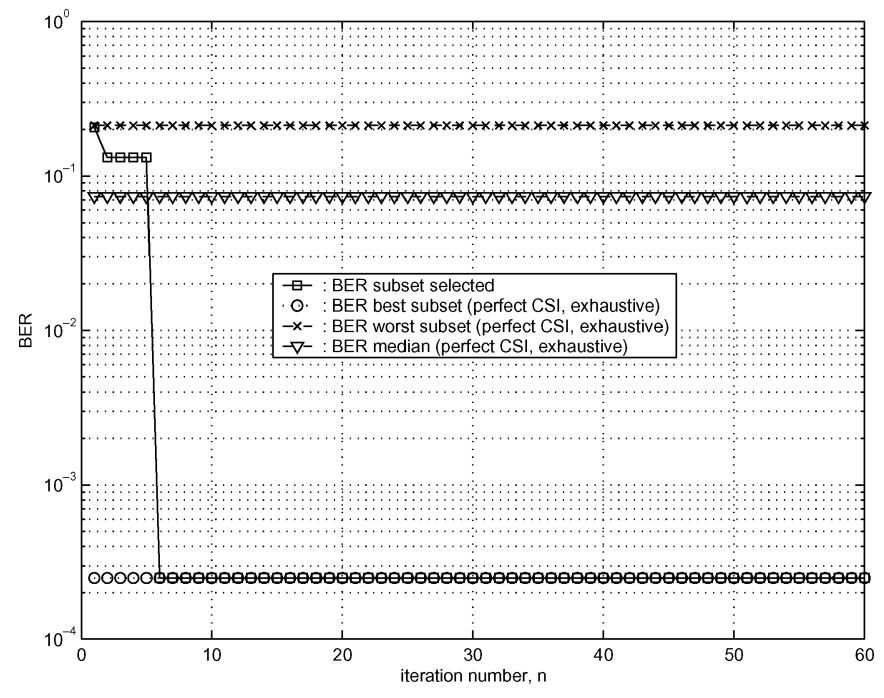

Fig. 8. Single run of Algorithm 1: BER of the of the chosen antenna subset versus iteration number $n$ in a system employing the ordered nulling (MMSE) and cancellation BLAST receiver.

algorithm converges to the optimal antenna subset. Moreover, it is observed that antenna selection at the receiver improves the BER by more than two orders of magnitude with respect to the median BER.

We now consider the average of 2000 runs of the algorithm over a new channel realization employing the ordered nulling and cancellation BLAST receiver. We set $\rho=9 \mathrm{~dB}$ and we use $T=6$ orthogonal training symbols to estimate the channel. Before starting the algorithm, long simulations are performed assuming perfect channel knowledge over all antenna configurations to find the exact BER associated with each antenna subset. We consider four different implementations of the algorithm depending on the length of the fake sequence $m$ and the $\rho$ used in the simulations: 1) the simulation to estimate the BER at every iteration of the algorithm is performed with the exact $\rho$ of the channel and $m=500$ fake symbols; 2 ) to reduce the complexity, the simulation is performed with the exact SNR of the channel $\rho=9 \mathrm{~dB}$ but with only $m=20$ fake symbols; 3 ) the SNR is reduced to $\rho=4 \mathrm{~dB}$ and $m=500$ fake symbols are employed; and 4) the SNR is reduced to $\rho=4 \mathrm{~dB}$ and only $m=20$ fake symbols are employed. In Fig. 9 , the average of the exact BER selected by the algorithm at each iteration is plotted. In the same figure, we show the BER of the best antenna subset and worst antenna subset, as well as the median BER among the 15 antenna configurations, found by exhaustive search. It is seen that the algorithm moves toward the optimal antenna configuration in the four cases considered. Comparing the performance of cases 1) and 2), we observe that 1) has a better convergence behavior because by using longer fake sequences, the estimate of the BER is less noisy. Comparing the performance of cases 1) and 4), we find that the behavior is very close although in 4) we have reduced the complexity by more than one order of magnitude. Comparing the performance of 2) and 4), we observe that although 2) uses the real $\rho$ of the channel to estimate the BER, the behavior is worse. This result is due to the fact that at very low values of the exact BER (i.e., high SNR) we cannot obtain a good estimate of the BER with only $m=20$

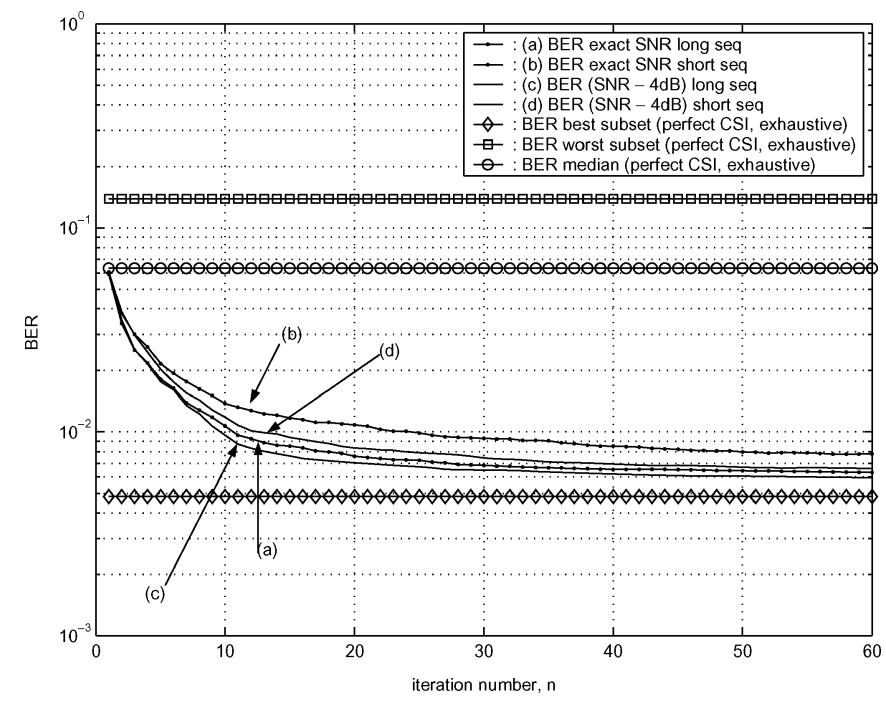

Fig. 9. Average of 2000 runs of the algorithm: Exact BER of the chosen antenna subset versus iteration number $n$ in a system employing the ordered nulling (MMSE) and cancellation BLAST receiver.

symbols. Moreover, we observe that case 3) has the best performance since with $m=500$ symbols we can have a better estimate of the BER when the SNR is $5 \mathrm{~dB}$. However, although not plotted in the figure, if the number $m$ of fake symbols became larger, the performance of 1) would become better than the one of 3). In summary, we can reduce the complexity without incurring in a convergence penalty by reducing the SNR of the simulations (assuming that the SNR difference is not large) and using a shorter sequence of fake symbols $m$.

\section{ADAPTiVe Algorithms FOR ANTENNA SELECTION IN TIME-VARYING CHANNELS}

In the previous section, we described discrete stochastic approximation algorithms for antenna selection in static MIMO channels. Now we consider nonstationary environments for which the optimum antenna subset takes on a time-varying form, $\omega^{*}[n] \in \Omega$, since the MIMO channel is time-varying. Consequently, the MIMO antenna selection algorithms should be able to track the best antenna subset if the variation of the channel is slow for tracking to be feasible. In general, any efficient adaptive solution in time-varying scenarios should include some form of recursive selection. The adaptive discrete stochastic approximation algorithms proposed in this section are directly applicable to any of the objective functions discussed in Section IV.

\section{A. Fixed Step-Size Discrete Stochastic Approximation Algorithm}

In the static channel environment discussed in the previous section, in order for the method to converge, it was necessary for the method to become progressively more and more conservative as the number of iterations grew. Consequently, a decreasing step size, $\mu[n]=1 / n$, was used, in order to avoid moving away from a promising point unless there was a strong evidence that the move will result in an improvement. In the time-varying case, we require a step size that permits moving away from a state as the optimal antenna subset changes [24]. 
Therefore, to track the optimal antenna subset, we replace the Adaptive filter for updating state occupation probabilities step in Algorithm 1 by

$$
\boldsymbol{\pi}[n+1]=\boldsymbol{\pi}[n]+\mu(\boldsymbol{D}[n+1]-\boldsymbol{\pi}[n])
$$

where $0<\mu \ll 1$. A fixed step size $\mu$ in (46) introduces an exponential forgetting factor of the past occupation probabilities and allows to track slowly time-varying optimal antenna subset $\omega^{*}[n]$. The same arguments can be used to extend the application of Algorithm 2 to time-varying channels by using a fixed step size $\mu$ in (15).

For $\boldsymbol{\pi}[n]$ being a probability vector (i.e., the elements add 1 and are nonnegative) the step size must satisfy $0<\mu \leq 1$. Note that $\mathbf{1}^{T}(\boldsymbol{D}[n+1]+\boldsymbol{\pi}[n])=0$ implying that $\mathbf{1}^{T} \boldsymbol{\pi}[n+1]=$ $\boldsymbol{1}^{T} \boldsymbol{\pi}[n]=1$. Expressing $(46)$ as $(1-\mu) \boldsymbol{\pi}[n]+\mu \boldsymbol{D}[n+1]$ we observe that the elements of $\boldsymbol{\pi}[n+1]$ are nonnegative, which proves that $\boldsymbol{\pi}$ is a probability vector.

Recall the inefficient brute force off-line approach in (9) and (10) for static channels. We showed that an exhaustive search over a large number of symbol blocks while optimal (as the number of estimates $N$ goes to infinity), is numerically very inefficient. Based on the off-line brute force approach, one obvious candidate for a time-varying channel is to do a brute force exhaustive search independently for every new symbol block. Actually, the brute force off-line search can be viewed as an extreme case of our recursive algorithm by setting a forgetting factor such that all the previous estimates are forgotten. That is, the exhaustive search without taking into account previous calculations of the objective function is a special case of our new algorithm with forgetting factor $\mu=1$. That is, all the objective function calculations using non promising antenna subsets would be completely wasted in the second symbol block. Thus, when the number of antenna subsets is large, this solution would be very inefficient, highly complex, and still, it would not converge to the optimal solution. Therefore, the key difference between the exhaustive search and the proposed recursive approach is that our proposed algorithm does not have a forgetting factor that completely disregards previous estimates. We refer to our recent paper [37] for a rigorous weak convergence analysis of our tracking algorithm.

It has been observed that time-varying channels modify the optimal antenna subset over the time although most of the antennas in the optimal antenna subset remain the same. Hence, in time-varying channels, we can modify the Sampling and Evaluation step in Algorithm 1 to select a candidate solution $\tilde{\omega}^{(n)}$ uniformly from $\Theta \backslash \omega^{(n)}$ where $\Theta$ is defined as the set of antenna subsets $\tilde{\omega}^{(n)} \in \Omega$ such that the distance $d\left(\tilde{\omega}^{(n)}, \omega^{(n)}\right)=D$, where we choose $D<\min \left(n_{R}, N_{R}-n_{R}\right)$.

Simulation Results: We demonstrate the tracking performance of this version of the algorithm under the maximum mutual information criterion in time-varying channels. We use (33) as an estimate of the objective function. We assume that each channel gain $h_{i, j}$ between a transmit and receive antenna remains constant over $\tau$ symbol block intervals (we assume that each block interval corresponds to one iteration of the

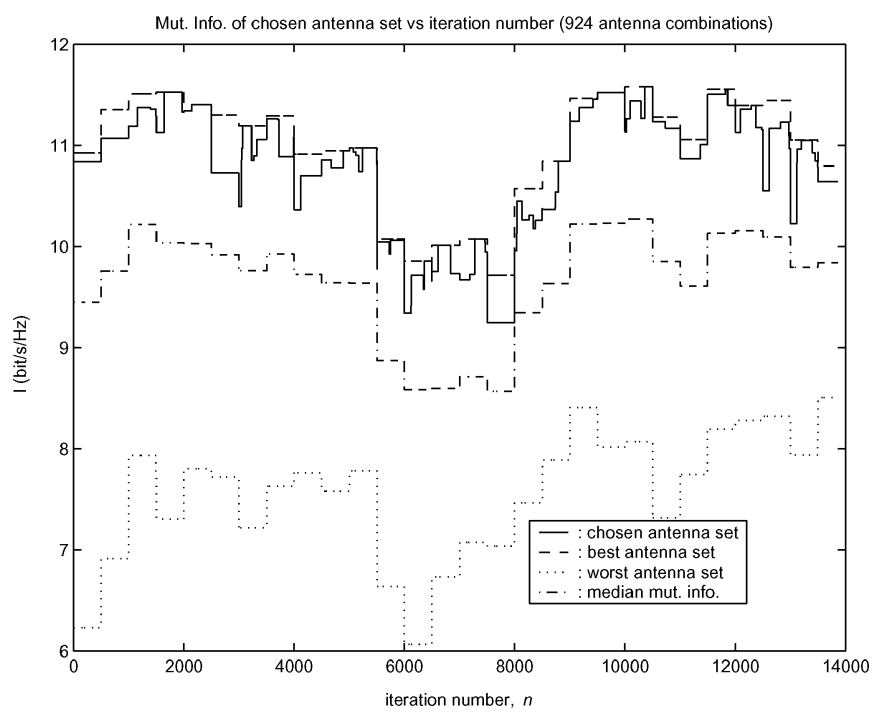

Fig. 10. Mutual information values of the chosen antenna subsets versus iteration number $n$ (fixed step-size).

algorithm) and follows a first order AR dynamics over $\tau$ written as

$$
\begin{array}{r}
h_{i, j}(t)=\alpha h_{i, j}(t-1)+\beta v_{i, j}(t) \\
i=1, \ldots, N_{R} \text { and } j=1, \ldots, N_{T}
\end{array}
$$

where $\alpha$ and $\beta$ are the fixed parameters of the model related through $\beta=\left(1-\alpha^{2}\right)^{1 / 2}$ and $v_{i, j} \sim \mathcal{N}_{c}(0,1)$. The parameter $\alpha$ can be related to the maximum Doppler frequency $f_{d}$ as $\alpha=$ $J_{0}\left(2 \pi f_{d} \tau T_{f}\right)$, where $J_{0}(\cdot)$ is the zeroth-order Bessel function of the first kind, and $T_{f}$ is the duration of one block interval. In the simulations, we set $\alpha=0.9, \tau=500$ and the constant step size $\mu=0.002$. We consider $N_{R}=12, n_{R}=6$ and $n_{T}=2$. We set $\rho=10 \mathrm{~dB}$ and we use the ML channel estimate with $T=6$ orthogonal training symbols to estimate the channel. It has also been observed that in most cases $d\left(\omega^{*}[n], \omega^{*}[n-\tau]\right) \leq$ 2 and therefore we set $D=2$. The tracking performance of the algorithm is shown in Fig. 10. The maximum, minimum and median values of the mutual information as a function of time are also shown. It is seen that the algorithm closely tracks the best antenna subset.

\section{B. Adaptive Step-Size Discrete Stochastic Approximation Algorithm}

In the previous version of the algorithm, the choice of the fixed step size $\mu$ has high influence in the performance of the algorithm. The faster the channel changes or the further away of the current subset estimate of the optimal antenna subset, the larger $\mu$ should be. On the other hand, the larger the effects of the observation noise or the closer we are from the optimal antenna subset, the smaller $\mu$ should be [27]. However, in practice, one does not know the dynamics of the channel in advance.

In this section we present a method to adaptively adjust the step size $\mu[n]$ as the algorithm evolves. In this way, at each iteration $n$, our stochastic approximation algorithm has two estimation problems to contend with. The first is the estimation of $\omega^{*}[n]$ and the second is the estimation of $\mu[n]$. Since the $\mu[n]$ is 
a continuous variable, we can use an adaptive algorithm similar to the gradient descent algorithm [7]. This underlying adaptive algorithm to adjust $\mu[n]$ would use estimates of the derivative of the mean square error with respect to the step size $\mu$. These ideas are based on [7] and [26] and have been further exploited in [24] and [25].

Within this new framework, the estate occupation probability vector depends on $\mu$. Denote the mean-square derivative $(\partial / \partial \mu) \boldsymbol{\pi}^{\mu}[n]$ by $\boldsymbol{J}^{\mu}[n]$, i.e.,

$$
\lim _{\Delta \rightarrow 0} E\left\{\left|\frac{\boldsymbol{\pi}^{\mu+\Delta}[n]-\boldsymbol{\pi}^{\mu}[n]}{\Delta}-\boldsymbol{J}^{\mu}[n]\right|^{2}\right\}=0 .
$$

Define the error

$$
\boldsymbol{e}^{\mu}[n]=\boldsymbol{D}[n+1]-\boldsymbol{\pi}^{\mu}[n]
$$

and differentiate the square of the error with respect $\mu$ as

$$
\frac{\partial}{\partial \mu}\left(\boldsymbol{e}^{\mu}[n] \boldsymbol{e}^{\mu}[n]^{T}\right)=-2\left(\boldsymbol{D}[n+1]-\boldsymbol{\pi}^{\mu}[n]\right)^{T} \boldsymbol{J}^{\mu}[n]
$$

Next, differentiating $\boldsymbol{\pi}[n+1]$ in (46) with respect to $\mu$ yields

$$
\boldsymbol{J}^{\mu}[n+1]=\boldsymbol{J}^{\mu}[n]-\mu \boldsymbol{J}^{\mu}[n]+\left(\boldsymbol{D}[n+1]-\boldsymbol{\pi}^{\mu}[n]\right)
$$

The proposed scheme aims to minimize the expectation of (49) by scaling $\mu[n]$ depending on the error in (49). The following adaptive step-size discrete stochastic approximation algorithm is adopted as a modification of Algorithm 1.

Algorithm 3 Adaptive Step-Size Discrete Stochastic Approximation Algorithm

Initialization, Sampling, and Acceptance: the same as Algorithm 1

Substitute the update of the state occupation probabilities by

$$
\begin{aligned}
e[n] & =\boldsymbol{D}[n+1]-\boldsymbol{\pi}[n], \\
\boldsymbol{\pi}[n+1] & =\boldsymbol{\pi}[n+1]+\mu[n] \boldsymbol{e}[n], \\
\mu[n+1] & =\left\{\mu[n]+\eta \boldsymbol{e}^{T}[n] \boldsymbol{J}[n]\right\}_{\mu_{-}}^{\mu_{+}} \\
\boldsymbol{J}[n+1] & =(1-\mu[n]) \boldsymbol{J}[n]+\boldsymbol{e}[n], \quad \boldsymbol{J}[0]=\mathbf{0} .
\end{aligned}
$$

Compute the maximum: the same as Algorithm 1

In the algorithm, $\eta$ denotes the learning rate. As $\eta$ decreases, the rate of adaptation decreases. If the learning rate $\eta=0$, then the algorithm reduces to the fixed step-size algorithm. $\{X\}_{\mu_{-}}^{\mu_{+}}$ denotes the projection of $X$ onto the interval $\left[\mu_{-}, \mu_{+}\right]$with $0<\mu_{-} \leq \mu_{+}$. For fast speed of tracking and good transient behavior, one seeks $\mu_{+}$as large as possible but not greater than the instability value. We note that the sequence $\mu[n]$ will not go to zero unless optimal antenna subset remains constant.

We point out that Algorithm 3 is composed of three parts: 1) A random search of a next candidate $\omega^{(n)}$ over $\Omega ; 2$ ) a continuous adaptive LMS algorithm which updates the step size $\mu[n]$; and 3) a discrete adaptive algorithm that updates the state probability vector $\pi[n]$, where the last two adaptive algorithms are crosscoupled. Assuming that there is a unique local minimum $\mu^{*}$ of

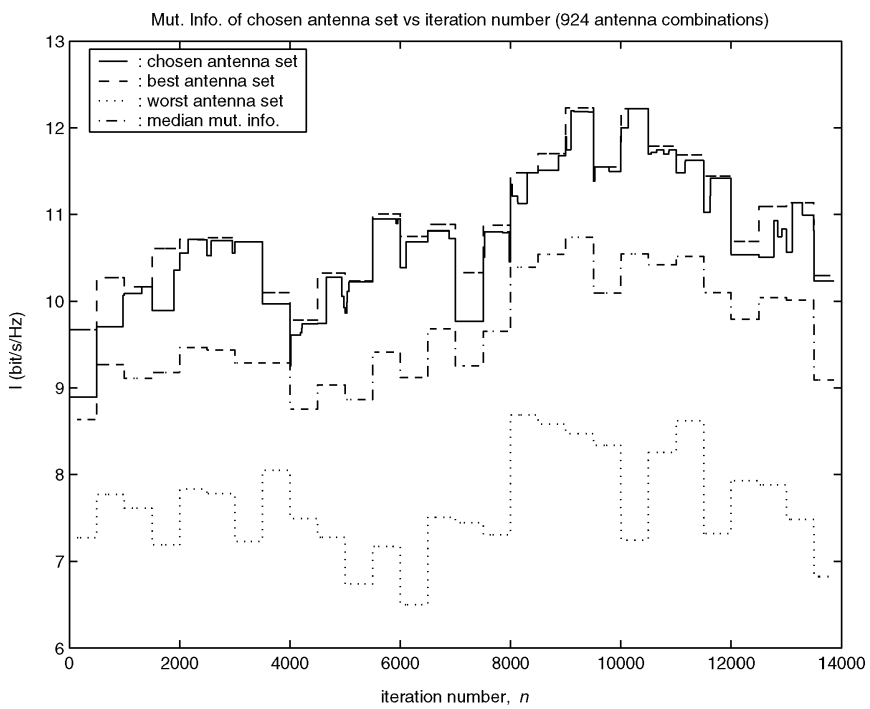

Fig. 11. Mutual information values of the chosen antenna subsets versus iteration number $n$ (adaptive step-size).

$E\left\{\left\|\boldsymbol{e}^{\mu}[n]\right\|^{2}\right\}$, it can be proven that $\mu[n]$ converges weakly to $\mu^{*}[24]$.

An interesting feature of the algorithm is that it does not assume anything about the dynamics of the problem. It self adapts to track the dynamics of the channel and consequently, the best antenna subset $\omega^{*}[n]$.

Simulations Results: To demonstrate the performance of this version of the algorithm, we consider the same system parameters as in Section V-A. The bounds for the step size are chosen as $\mu_{-}=0$ and $\mu_{+}=0.003$ and the learning rate is set to $\nu=$ 0.0005 . We restrict the candidate solution to antenna subsets with $D=2$. Fig. 11 shows the performance of the algorithm. The maximum, minimum and median values of the mutual information as a function of time are also shown for comparison. It is seen that the adaptive step-size algorithm has a better tracking performance than the constant step-size algorithm.

\section{CONCLUSIONS}

We have developed MIMO antenna selection algorithms based on various performance criteria in situations where only noisy estimates of the channels are available. The proposed techniques are based on the discrete stochastic approximation algorithms found in the recent operations research literature, which generate a sequence of antenna subsets where each new subset is obtained from the previous one by taking a small step in a good direction toward the global optimizer. One salient feature of the proposed approach is that no closed-form expression for the objective function is needed and only an estimate of it is sufficient. Therefore, the algorithm is able to choose the antenna subset that minimize the bit, symbol or frame error rate, under any MIMO techniques (e.g., BLAST, space-time coding) and any receiver detection methods.

We have also developed antenna selection algorithms for time-varying scenarios where the optimal antenna subset is 
slowly varying. By employing the constant or adaptive step-size discrete stochastic approximation algorithms, the time-varying optimal antenna configuration can be closely tracked. Finally, we have provided extensive simulation results to demonstrate the performance of these new MIMO antenna selection algorithms under various selection criteria.

\section{APPENDIX A}

\section{UNBIASED ESTIMATE OF $\operatorname{det}(\cdot)$}

Obtain an estimate of

$$
\operatorname{det}\left(\boldsymbol{I}_{n_{T}}+\frac{\rho}{n_{T}} \boldsymbol{H}^{H}[\omega] \boldsymbol{H}[\omega]\right)
$$

using

$$
\phi[n, \omega]=\operatorname{det}\left(\boldsymbol{I}_{n_{T}}+\frac{\rho}{n_{T}} \hat{\boldsymbol{H}}_{1}^{H}[n, \omega] \hat{\boldsymbol{H}}_{2}[n, \omega]\right)
$$

where the channel estimates $\hat{\boldsymbol{H}}_{1}[n, \omega]$ and $\hat{\boldsymbol{H}}_{2}[n, \omega]$ are obtained from independent training blocks. We consider the case in which $\hat{\boldsymbol{H}}_{1}[n, \omega]$ and $\hat{\boldsymbol{H}}_{2}[n, \omega]$ satisfy (6).

Theorem 4: With $\phi[n, \omega]$ computed according to (54), the estimate of the determinant in (53) is unbiased.

Proof: For convenience, define

$$
\begin{aligned}
\boldsymbol{M}[\omega] & =\boldsymbol{I}_{n_{T}}+\frac{\rho}{n_{T}} \boldsymbol{H}^{H}[\omega] \boldsymbol{H}[\omega] \\
\hat{\boldsymbol{M}}[n, \omega] & =\boldsymbol{I}_{n_{T}}+\frac{\rho}{n_{T}} \hat{\boldsymbol{H}}_{1}^{H}[n, \omega] \hat{\boldsymbol{H}}_{2}[n, \omega]
\end{aligned}
$$

and denote the elements of $\hat{\boldsymbol{M}}[n, \omega]$ as $\hat{m}_{i, j}$.

Consider (55). Since $\hat{\boldsymbol{H}}_{1}[n, \boldsymbol{\omega}]$ and $\hat{\boldsymbol{H}}_{2}[n, \omega]$ are statistically independent samples, clearly $\hat{M}[n, \omega]$ is an unbiased estimator of $\boldsymbol{M}[\omega]$. Now consider $\operatorname{det}(\hat{\boldsymbol{M}}[n, \omega])$. From $[21$, p. 8]

$$
\operatorname{det}(\hat{\boldsymbol{M}}[n, \omega])=\sum_{\sigma} \operatorname{sign}(\sigma) \prod_{i=1}^{n_{T}} \hat{m}_{i, \sigma(i)}
$$

where the sum runs over all $n_{T}$ ! permutations $\sigma$ of the $n_{T}$ items $\left\{1, \ldots, n_{T}\right\}$ and $\operatorname{sign}(\sigma)$ is +1 or -1 . Omitting the sign, each term in the summation is of the form

$$
\hat{m}_{1, \sigma(1)} \hat{m}_{2, \sigma(2)} \ldots \hat{m}_{n_{T}, \sigma\left(n_{T}\right)} \text {. }
$$

Thus, each term in the summation involves the product of elements of $\hat{\boldsymbol{M}}[n, \omega]$ from different rows and columns.

Next, due to the independence assumption in (6), it follows that for the matrix $\hat{M}[n, \omega]$, the elements $\hat{m}_{i, j}$ and $\hat{m}_{p, q}$ are independent for $i \neq p$ and $j \neq q$, i.e., elements of $\hat{M}[n, \omega]$ from distinct rows and columns are statistically independent. Hence $\hat{m}_{1, \sigma(1)}, \hat{m}_{1, \sigma(1)}, \ldots, \hat{m}_{n_{T}, \sigma\left(n_{T}\right)}$ are statistically independent with zero mean which implies that $\operatorname{det}(\hat{\boldsymbol{M}}[n, \omega])$ is an unbiased sample of $\operatorname{det}(\boldsymbol{M}[\omega])$ and satisfies

$$
\operatorname{det}(\hat{\boldsymbol{M}}[n, \omega])=\operatorname{det}(\boldsymbol{M}[\omega])+v[n, \omega]
$$

where $v[n, \omega]$ is a zero mean random variable.

\section{REFERENCES}

[1] S. M. Alamouti, "A simple transmit diversity technique for wireless communications," IEEE J. Select. Areas Commun., vol. 16, no. 8, pp. 1451-1458, Oct. 1998

[2] M. Alrefaei and S. Andradottir, "A simulated annealing algorithm with constant temperature for discrete stochastic optimization," Manag. Sci., vol. 45, no. 5, pp. 748-764, 1999.
[3] S. Andradottir, "A global search method for discrete stochastic optimization," SIAM J. Optimiz., vol. 6, no. 2, pp. 513-530, May 1996.

[4] —, "Accelerating the convergence of random search methods for discrete stochastic optimization," ACM Trans. Modeling and Compu. Simul., vol. 9, no. 4, pp. 349-380, Oct. 1999.

[5] C. R. N. Athaudage and V. Krishnamurthy, "A low complexity timing and frequency synchronization algorithm for OFDM systems," in Proc. IEEE Globecom, Taipei, Taiwan, Nov. 2002.

[6] I. Bahceci, T. M. Duman, and Y. Altunbasak, "Antenna selection for multiple-antenna transmission systems: performance analysis and code construction," IEEE Trans. Inform. Theory, vol. 49, no. 10, pp. 2669-2681, Oct. 2003.

[7] A. Benveniste, M. Metivier, and P. Priouret, Adaptive Algorithms and Stochastic Approximation. Berlin/New York: Springer Verlag, 1990.

[8] T. H. de Mello, "Variable-sample methods for stochastic optimization," ACM Trans. Modeling and Computer Simulation, vol. 13, no. 2, pp. 108-133, Apr. 2003.

[9] G. J. Foschini and M. J. Gans, "On the limits of wireless communications in a fading environment when using multiple antennas," Wireless Personal Commun., vol. 6, no. 3, pp. 311-335, 1998.

[10] M. Gharavi-Alkhansari and A. B. Gershman, "Fast antenna subset selection in MIMO systems," IEEE Trans. Signal Process., vol. 52, no. 2, pp. 339-347, Feb. 2004.

[11] P. W. Glynn and W. Whitt, "The asymptotic efficiency of simulation estimators," Oper. Res., vol. 40, no. 3, May-Jun. 1992.

[12] G. D. Golden, G. J. Foschini, R. A. Valenzuela, and P. W. Wolniansky, "Detection algorithm and initial laboratory results using the V-BLAST space-time communication architecture," Electron. Lett., vol. 35, no. 1, pp. 14-15, Jan. 1999.

[13] D. Gore and A. Paulraj, "Statistical MIMO antenna sub-set selection with space-time coding," in Proc. IEEE Int. Conf. Commun. (ICC), New York, Apr. 2002.

[14] D. A. Gore and A. J. Paulraj, "MIMO antenna subset selection with space-time coding," IEEE Trans. Signal Process., vol. 50, no. 10, pp. 2580-2588, Oct. 2002.

[15] A. Gorokhov, "Antenna selection algorithms for MEA transmission systems," in Proc. IEEE Int. Conf. Acoust. Speech, Sig. Processing (ICASSP), Orlando, FL, May 2002.

[16] A. Gorokhov, D. A. Gore, and A. J. Paulraj, "Receive antenna selection for MIMO spatial multiplexing: theory and algorithms," IEEE Trans. Signal Process., vol. 51, no. 11, pp. 2796-2807, Nov. 2003.

[17] I. S. Gradshteyn and I. M. Ryzhik, Table of Integrals, Series, and Products, 5th ed. Orlando, FL: Academic, 1994.

[18] R. Heath and A. Paulraj, "Antenna selection for spatial multiplexing systems based on minimum error rate," in Proc. IEEE Int. Conf. Commun. (ICC), Helsinki, Finland, Jun. 2001.

[19] R. W. Heath and A. Paulraj, "Switching between spatial multiplexing and transmit diversity based on constellation distance," in Proc. Annu. Allerton Conf. Communications, Computing, and Control, Monticello, IL, Oct. 2000.

[20] Y. Hochberg and A. C. Tamhane, Multiple Comparison Procedures. New York: Wiley, 1987.

[21] R. A. Horn and C. R. Johnson, Matrix Analysis. Cambridge, U.K.: Cambridge Univ. Press, 1985.

[22] M. Kang and M. S. Alouini, "Capacity of MIMO Rician channels," IEEE Trans. Wireless Commun., 2004, submitted for publication.

[23] N. Kong and L. B. Milstein, "Average SNR of a generalized diversity selection combining scheme," IEEE Commun. Lett., vol. 3, no. 3, pp. 57-59, Mar. 1999.

[24] V. Krishnamurthy, X. Wang, and G. Yin, "Spreading code optimization and adaptation in CDMA via discrete stochastic approximation," IEEE Trans. Inform. Theory, vol. 50, no. 9, pp. 1927-1949, Aug. 2004.

[25] V. Krishnamurthy, G. Yin, and S. Singh, "Adaptive step size algorithms for blind interference suppression in DS/CDMA systems," IEEE Trans. Signal Process., vol. 49, no. 1, pp. 190-201, Jan. 2001.

[26] H. J. Kushner and J. Yang, "Analysis of adaptive step-size SA algorithms for parameter tracking," IEEE Trans. Autom. Control, vol. 40, no. 8, pp. 1403-1410, Aug. 1995.

[27] H. J. Kushner and G. Yin, Stochastic Approximation Algorithms and Applications. New York: Springer-Verlag, 1997.

[28] T. L. Marzetta, "BLAST training: estimation channel characteristics for high-capacity space-time wireless," in Proc. 37th Annu. Allerton Conf. Communications, Computing, \& Control, Monticello, IL, Sep. 1999.

[29] A. F. Molisch, M. Z. Win, and J. H. Winters, "Capacity of MIMO systems with antenna selection," in Proc. IEEE Int. Conf. Communication (ICC), Helsinki, Finland, Jun. 2001. 
[30] R. Nabar, D. Gore, and A. Paulraj, "Optimal selection and use of transmit antennas in wireless systems," in Proc. Int. Conf. Telecommun. (ICT), Acapulco, Mexico, May 2000.

[31] H. Sampath, P. Stoica, and A. J. Paulraj, "Generalized linear precoder and decoder design for MIMO channels using the weighted MMSE criterion," IEEE Trans. Commun., vol. 49, no. 12, pp. 2198-2206, Dec. 2001.

[32] L. Shi and S. Olafsson, "An integrated framework for deterministic and stochastic optimization," in Proc. Winter Simulation Conf., 1997, pp. 395-401.

[33] V. Tarokh, H. Jafarkhami, and A. R. Calderbank, "Space-time block codes from orthogonal designs," IEEE Trans. Inform. Theory., vol. 45, no. 5, pp. 1456-1467, Jul. 1999.

[34] I. E. Telatar, "Capacity of multi-antenna Gaussian channels," Euro. Trans. Telecommun., vol. 10, no. 6, pp. 585-595, Nov. 1999.

[35] M. Z. Win and J. H. Winters, "Analysis of hybrid selection/maximalratio combining in rayleigh fading," IEEE Trans. Commun., vol. 47, no. 12, pp. 1773-1776, Nov. 1999.

[36] D. Yan and H. Mukai, "Stochastic discrete optimization," SIAM J. Control and Optimiz., vol. 30, no. 3, pp. 594-612, Oct. 1992.

[37] G. Yin, V. Krishnamurthy, and C. Ion, "Regime switching stochastic approximation algorithms with application to adaptive discrete stochastic optimization," SIAM J. Opt., vol. 14, no. 4, pp. 117-1215, 2004.

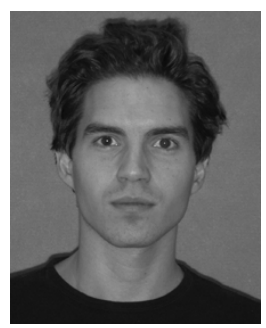

Inaki Berenguer received the Master's and Ph.D. degrees in engineering from Cambridge University, Cambridge, U.K., in 2002 and 2004, respectively, and was a two-year Fulbright scholar at Columbia University, New York.

From July 1999 to January 2001, he was with Hewlett Packard, Brussels, Belgium. In January 2001, he joined the Wireless Division of STMicroelectronics, San Diego, CA, where he worked on a W-CDMA project until August 2001. In the summer of 2004 he worked for Intel Corporation, designing advanced signal processing algorithms for video processors, and from September through December 2004 and in the summer of 2005, he was a Research Associate at NEC Laboratories America, Princeton, NJ. He is currently a Research Fellow of The Communications Innovations Institute, Cambridge, U.K., funded by the Cambridge-MIT Institute (CMI). He is also the recipient of the British Council, La Caixa, Cambridge-MIT Institute, and Leonardo Da Vinci fellowships.

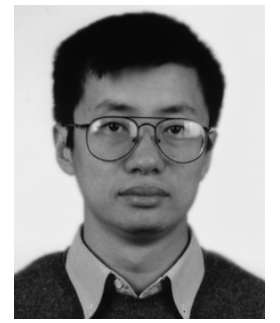

Xiaodong Wang received the B.S. degree in electrical engineering and applied mathematics (with highest honors) from Shanghai Jiao Tong University, Shanghai, China, in 1992, the M.S. degree in electrical and computer engineering from Purdue University, West Lafayette, IN, in 1995, and the $\mathrm{Ph} . \mathrm{D}$. degree in electrical engineering from Princeton University, Princeton, NJ, in 1998

From July 1998 to December 2001, he was an Assistant Professor in the Department of Electrical Engineering, Texas A\&M University, College Station. In January 2002, he joined the faculty of the Department of Electrical Engineering, Columbia University, New York. His research interests fall in the general areas of computing, signal processing and communications. He has worked in the areas of digital communications, digital signal processing, parallel and distributed computing, nanoelectronics and bioinformatics, and has published extensively in these areas. Among his publications is a recent book entitled Wireless Communication Systems: Advanced Techniques for Signal Reception (Upper Saddle River, NJ: Prentice-Hall, 2003). His current research interests include wireless communications, Monte Carlo-based statistical signal processing, and genomic signal processing.

Dr. Wang received the 1999 National Science Foundation CAREER Award and the 2001 IEEE Communications Society and Information Theory Society Joint Paper Award. He currently serves as an Associate Editor for the IEEE TRANSACTIONS ON COMMUNICATIONS, the IEEE TRANSACTIONS ON WIRELESS COMmUNiCATIONS, the IEEE TRANSACTIONS ON SignAl PROCESSING, and the IEEE TRANSACTIONS ON INFORMATION THEORY.

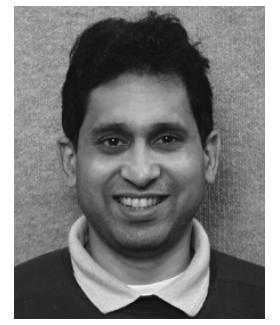

Vikram Krishnamurthy (F'05) was born in 1966 He received the Bachelor's degree from the University of Auckland, Auckland, New Zealand, in 1988 and the Ph.D. degree from the Australian National University, Canberra, in 1992.

$\mathrm{He}$ is currently a Professor and Canada Research Chair at the Department of Electrical Engineering, University of British Columbia, Vancouver, BC, Canada. Prior to 2002, he was a Chaired Professor at the Department of Electrical and Electronic Engineering, University of Melbourne, Parkville, Australia, where he also served as Deputy Head of department. His current research interests include ion channels and biological nanotubes, networked sensor scheduling and control, statistical signal processing, nonlinear filtering, and wireless telecommunications.

Dr. Krishnamurthy is currently an associate editor for IEEE TRANSACTIONS on Signal PRocessing, IEEE TRANSACtions ON AEROSPACE AND ELECTRONIC SYSTEMS, and SYSTEMS AND CONTROL LETTERS. He is guest editor of a special issue of IEEE TRANSACTIONS ON NANOBIOSCIENCE, March 2005 on bio-nanotubes. He currently serves on the Signal Processing Theory and Methods (SPTM) Technical Committee of the IEEE Signal Processing Society, and the International Federation of Automatic Control (IFAC) Technical Committee on Modeling, Identification and Signal Processing. He has served on the technical program committee of several conferences in signal processing, telecommunications and control. 